\title{
Reconfiguration Strategies with Composite Data Physicalizations
}

\author{
Kim Sauvé \\ kim.sauve@lancaster.ac.uk \\ Lancaster University \\ United Kingdom
}

\author{
David Verweij \\ d.verweij2@newcastle.ac.uk \\ Newcastle University \\ United Kingdom
}

\author{
Jason Alexander \\ jma73@bath.ac.uk \\ University of Bath \\ United Kingdom
}

\author{
Steven Houben \\ s.houben@lancaster.ac.uk \\ Lancaster University \\ United Kingdom
}
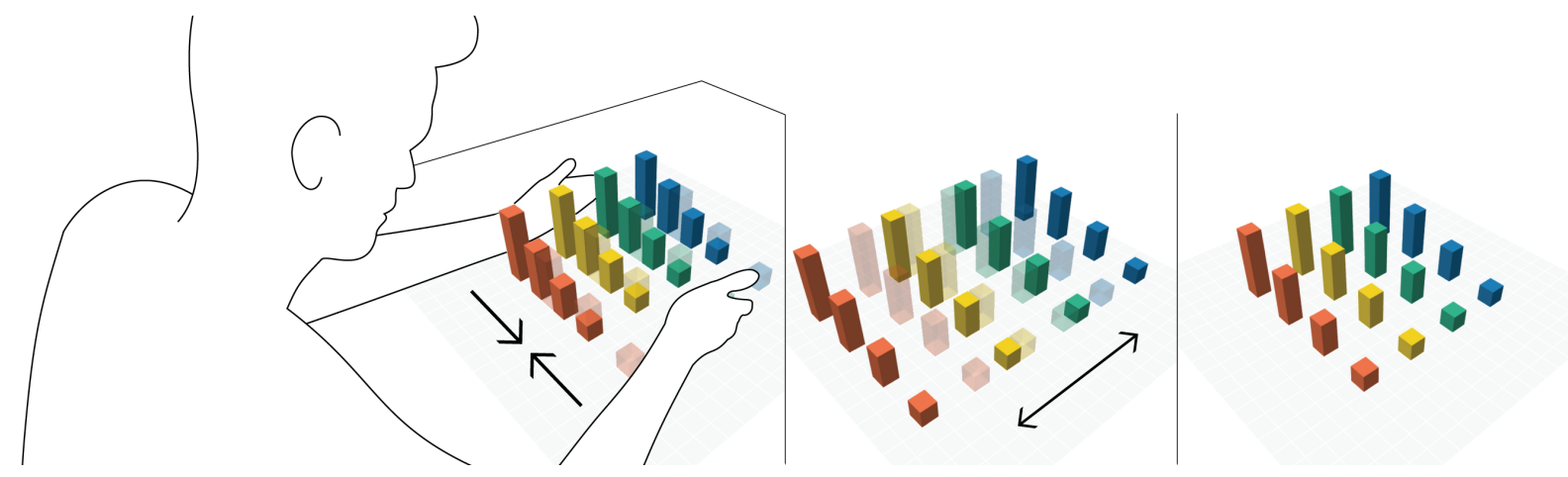

Figure 1: A digital render of one of the exemplar physicalizations (phys1, right), colors depict identified clusters by the participant. Various reconfiguration strategies were observed, including the increase of cohesion within clusters (left) and the increase of separation between clusters (middle).

\begin{abstract}
Composite data physicalizations allow for the physical reconfiguration of data points, creating new opportunities for interaction and engagement. However, there is a lack of understanding of people's strategies and behaviors when directly manipulating physical data objects. In this paper, we systematically characterize different reconfiguration strategies using six exemplar physicalizations. We asked 20 participants to reorganize these exemplars with two levels of restriction: changing a single data object versus changing multiple data objects. Our findings show that there were two main reconfiguration strategies used: changes in proximity and changes in atomic orientation. We further characterize these using concrete examples of participant actions in relation to the structure of the physicalizations. We contribute an overview of reconfiguration strategies, which informs the design of future manually reconfigurable and dynamic composite physicalizations.
\end{abstract}

\section{CCS CONCEPTS}

\section{- Human-centered computing $\rightarrow$ User studies; Visualization.}

\footnotetext{
Permission to make digital or hard copies of all or part of this work for personal or classroom use is granted without fee provided that copies are not made or distributed for profit or commercial advantage and that copies bear this notice and the full citation on the first page. Copyrights for components of this work owned by others than the author(s) must be honored. Abstracting with credit is permitted. To copy otherwise, or republish, to post on servers or to redistribute to lists, requires prior specific permission and/or a fee. Request permissions from permissions@acm.org.

CHI '21, May 8-13, 2021, Yokohama, Japan

(C) 2021 Copyright held by the owner/author(s). Publication rights licensed to ACM. ACM ISBN 978-1-4503-8096-6/21/05 ...\$15.00

https://doi.org/10.1145/3411764.3445746
}

\section{KEYWORDS}

Data Physicalization, Physical Visualization, Composite Data Physicalization, Reconfiguration

ACM Reference Format:

Kim Sauvé, David Verweij, Jason Alexander, and Steven Houben. 2021. Reconfiguration Strategies with Composite Data Physicalizations. In $\mathrm{CHI}$ Conference on Human Factors in Computing Systems (CHI '21), May 8-13, 2021, Yokohama, fapan. ACM, New York, NY, USA, 18 pages. https://doi.org/ $10.1145 / 3411764.3445746$

\section{INTRODUCTION}

Physicalizations are physical artefacts whose "geometry or material properties encode data" [17], allowing users to perceive information in a tangible or physical form factor. Fundamentally, physicalizations afford physical interaction (touching, grabbing, pushing, etc.) and some form of reconfiguration as their physicality encourages people to interact with their tangible elements. In many instances (e.g. $[10,14,22])$ these physicalizations support data curation and input where users can physically rearrange data points. Our research is concerned with a better understanding of the practices, strategies, and approaches people take when interacting with physicalizations.

There are many examples of physicalizations where direct interaction with the data forms their core modus operandi [14-16, 23, 34]. Following the work of Le Goc et al., we focus specifically on composite physicalizations which consist of "multiple elements whose typology can be reconfigured or can reconfigure itself" [23]. Composite physicalizations thus allow manual (through user input) or automatic (through machine actuation) updates of the location and orientation of the data objects while keeping the basic building blocks 
internally consistent. Whilst interaction with composite physicalizations involves both physical and computational elements, only the physical elements dictate the complexity of interactions possible. Hence, when designing these systems, the level of granularity, degree of manipulability and level of actuation [23] of the overall composite physicalization depends on the number of physical elements involved and the extent to which these can be rearranged or can rearrange themselves (i.e. without human intervention).

There are three main categories of composite physicalizations. (1) Static composite physicalizations allow the user to manually reconfigure predetermined 'buckets' of data points (i.e. per category or year), with the physicalization unable to reconfigure itself [16, 19, 32]. For example, Jansen et al. [16] investigated how users interpreted rows of physical bar charts that could be manually rearranged. (2) Constructive visualizations [13] allow the free reconfiguration of non-actuated token-based composite physicalizations [10, 14, 35, 37]; instead of manipulating 'buckets' of data, users manually rearrange individual datapoints. For example, Huron et al. [14] investigated the use of physical tokens as a data authoring tool for non-experts. (3) Shape-changing interfaces [28] support interaction with dynamic composite physicalizations which, due to their ability to actuate, can respond to interaction as well as initiate changes themselves [5, 9, 22, 24, 34]. For example, Taher et al. [34] investigated the use of automated physical bar charts to explore and present exemplar data. All three approaches support the organization of data by direct physical manipulation, whether or not it is by comparing predesigned 'buckets' of data, constructing them with data points from scratch, or exploring them dynamically.

The unique affordances and characteristics of physicalizations make them distinct from $2 \mathrm{D}$ visualizations, unlocking novel humandata interaction approaches. For example, their three-dimensionality allows for observation from multiple angles and their tangibility supports active manipulation using everyday motor skills. Physicalizations also allow for social interactions around them that can facilitate collaboration [17]. However, we still lack an understanding of how users approach and interact with composite physicalizations and the facets of their distinct qualities. This includes the perception of size for different physical shapes [16], the effect of user orientation on the perception of physical information [29], and the interweaving of the material and social aspects of tangible interaction [12]. In addition, shape-changing physicalizations are currently bound to the limitations of technology for their implementation and often explored and studied in those terms. This means the study of users' interactions with these systems is currently restricted to technology dictated interactions, potentially conflicting with users' preferred or spontaneous interaction strategies. As such, we aim to further develop our understanding of approaches to, and interactions with, composite physicalizations. This will inform how we might better support users' approaches to reorganizing data in physical 3D space. Our work aims to investigate users' strategies when interacting with physical data points, e.g., in relation to each other, the canvas, and to themselves. In doing so, we can start to understand people's spontaneous reconfiguration strategies, regardless of any technological limitations or recognition biases the possible pre-existing association one can have with a dataset due to their prior knowledge, occupation, etc. - and with a higher degree of interaction possibilities.
In response to the highlighted gaps in understanding users and their interaction with physicalizations [12, 16, 29], our work focuses on interaction with composite bar chart physicalizations, to inform the next generation of interactive dynamic composite physicalizations. We conducted an experiment with six abstract exemplar physicalizations, informed by prior work on the well-known physical $3 \mathrm{D}$ bar charts $[5,7,9,34]$, to observe technologically unconstrained direct physical manipulation. We asked 20 participants to use any approach to reconfiguration to reorganize pre-identified clusters of data objects. Our key finding is the detailed breakdown of two main user strategies found for reorganizing physicalizations: changes in proximity, where objects are relocated in the same plane, and changes in atomic orientation, where objects are rotated. While these two strategies dominated and prevailed in our findings, they are not mutually exclusive and are complementary to other strategies, including the swapping and removal of data objects. Our contribution lies in our observation of the (dis)similarity of their use across different (exemplar) physicalizations and degree of user restrictions. We detail these (dis)similarities per physicalization, across different physicalizations, and two degrees of user restriction to reflect on generalisability across systems of different interaction possibilities. This contributes a first characterization of user strategies when reorganizing clustered data objects that are part of a larger physicalization. The real-world implication of this work is that, for a dynamic composite physicalization to allow for interaction, it should take into account these strategies in the interaction design, data presentation, and actuation mechanisms of the system.

\section{BACKGROUND}

Data Physicalizations are the physical analogy of Data Visualizations, encoding data into physical geometry and/or material properties [17]. Physical interaction with data can increase user engagement, facilitate understanding and learning, and make data more accessible [17]. Prior work on interaction with physicalizations demonstrated different interaction techniques to systems ranging from static (e.g. [16]) to fully actuated representations (e.g. [34]). These include the manual re-arrangement of static data columns or data points to organize exemplar data $[14,16]$, stacking physical tokens to construct individual data points [10], performing gestures in the air to control data filtering of dynamic physical bar charts [33] or pushing/pulling individual bars of a bar chart to change a data point's value in a linear manner [34].

\subsection{Composite data physicalizations}

Prior work studied specific underlying elements of the reconfiguration of physicalizations. Jansen et al. [18] have shown that the perception of size for physical bars is consistent with 2D visualizations, yet different for physical spheres. Sauvé et al. [29] found that the perception of physical information is in general directly influenced by user orientation to the canvas. Taher et al. [34] and Everitt et al. [7] found that participants mainly interacted with the most accessible physical bars along the edges of a system's grid, illustrating how the technical implementation can influence interaction. Lastly, Jansen et al. [16] found that touching a physicalization can be a cognitive aid for memory. 
In our work we focus on interaction with composite physicalizations [23] - a subset of physicalizations that allow for the physical reconfiguration of their elements, manually by the user and/or by automated actuation. These types of physicalizations offer promising opportunities for data curation and input, but equally raise challenges for interactive systems to support direct physical manipulations. Further, our current understanding of the implications of user reconfiguration of data is limited: such rearrangement effects data perception, integrity, discovery, and interpretation.

\subsection{Static composite physicalizations}

Static composite physicalizations allow the user to manually reconfigure predetermined 'buckets' of data points that cannot reconfigure themselves. Examples are work using re-arrangeable 3D bar charts [16], but also more expressive shapes to foster reflection [19, 32]. For example, SweatAtoms [19] and Activity Sculptures [32] use 3D printed objects to visualize activity data to allow users to appropriate and reflect on personal data.

\subsection{Constructive visualization}

Constructive visualization [13] supports the free reconfiguration of non-actuated token-based physical data representations. In other words, the physicalization is constructed by placing building blocks (tokens) on a blank 'canvas'. This supports data authoring through the reconfiguration of physical tokens, for example by stacking and/or changing the spatial relations in a plane. These interactions allow the construction and curation of data from scratch, but are constrained by components such as token unit, token grammar and assembly model [13]. Still, how people make use of space and perform spatial organization can be informative for the interaction with dynamic composite physicalizations. It is understood that the construction of physicalizations results in an interrelation principle [37], as moving physical elements influences multiple parameters of the visualization pipeline at once. For example, the decision to place physical objects in the canvas - loading data [37] - simultaneously requires the user to think about where to put the object in relation to other data objects - visual mapping [37] and in relation to the canvas - presentation mapping [37]. Example works make use of tangible tiles $[8,14,37]$ but also more complex token grammars such as Cairn [10] for situated data collection of a maker community, or more freeform tokens from household objects for the creation of personal physicalizations in the home [35].

\subsection{Shape-changing interfaces}

Shape-changing interfaces $[1,28]$ support interaction with dynamic composite physicalizations. For shape-changing interfaces, interaction often occurs by touching supplementary menu panels [7] or by pushing and pulling the actuated bars directly [34]. These interactions most often allow for navigation and exploration of data. Examples include automated 3D physical bars in a fixed grid [5, 9, 24, 34], but also freely re-arrangeable systems such as Zooids [22]. However, as these technologies are still not mature, it can be difficult to understand complex interaction that is not inherently constrained by the design goals of the system, the path of least resistance of the application, and frequently also the limitations of the technology.

\section{RATIONALE FOR METHODOLOGY}

While we are starting to build clear insights into how physicalizations can be constructed using emerging technologies $[9,24,33]$ and how they can encode data in specific application domains [7, 26, 31], there is currently little empirical work that examines the underlying mechanisms through which people interact with physicalizations [29]. We argue that understanding the underlying principles of perception and interaction with physicalizations are a necessary step to be able to design, build and research effective and consistent physicalizations.

Prior work demonstrates that the perception of and interaction with visualizations can be successfully studied in isolation [6]. Examples in the realm of physicalizations include the perception of physical size [18], the influence of orientation on perception [29] and the interaction with physical data points [14]. These studies use abstract non-interactive 'data-agnostic' apparatuses to enable a systematic and principled approach that leads to novel insights that generalize to a wide range of systems and applications. However, there are currently no studies that elucidate the underlying principles and strategies of how people interact with and reconfigure physical information.

In this work, we adopt a similar approach - using methodologies and apparatus from static physicalization and constructive visualization - that enables us to study reconfiguration strategies using abstract data points that adhere to the rules of Gestalt [21]. This allows us to design the layouts of exemplar physicalizations in such a way that they are not based on a single dataset, but adhere to relational properties of objects and the visual perception of space (as per Gestalt) that forms the foundational theory for any physical representation. Gestalt is important for data visualization and physicalization since it is concerned with how the human brain perceives information and what perceptual properties are easier to interpret than others. To design informative physicalizations, that are effective in communicating information through physical elements, it is important to adhere to these fundamental principles.

Our approach draws on prior work from the field of cognitive science on the use of physical space and clustering of physical (data) objects. Specifically, the proposed classification of Kirsh [20] explains that rearrangement of the position of physical (data) objects can serve three main purposes: (i) spatial arrangements that simplify choice; (ii) spatial arrangements that simplify perception; (iii) and spatial dynamics that simplify internal computation. To give an example, people offload mental effort into physical space by rearranging objects to simplify choice or to try out alternatives [20]. Hence, interaction with physical data objects goes beyond pure 'data interpretation' tasks but engages cognition and perception of physical space [20,29].

While our work builds principally on work in the field of physicalizations [17], this work is dominated by research on technology (new forms of implementing actuated physicalizations), and specific domains (implementing datasets in physicalizations). Because of the systematic lack of more principled and fundamental work, we build further on prior work in $2 \mathrm{D}$ visualization to inform our study setup. Our study operationalizes pre-attentive properties (e.g. visual salience) [36, Chapter 5] and Gestalt principles [36, Chapter 6] in the design of the stimuli. In our findings, we draw parallels 


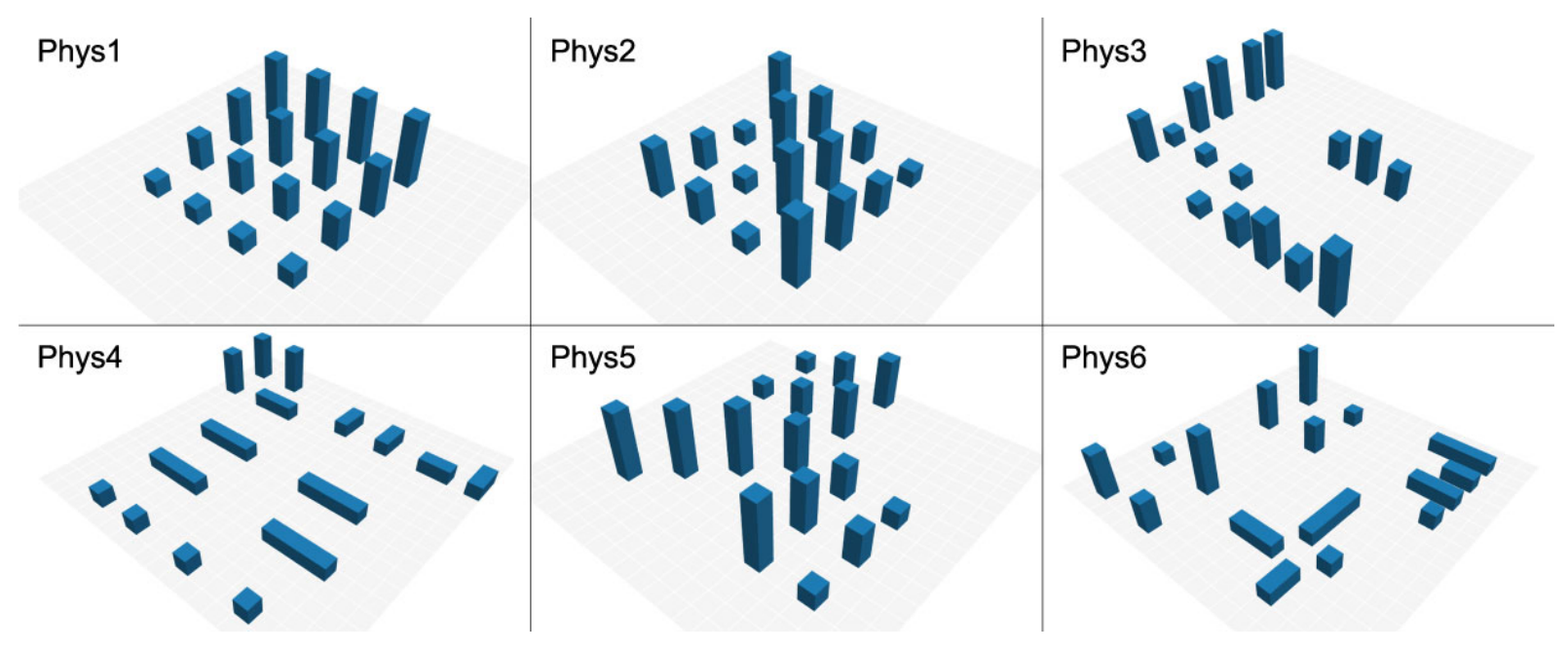

Figure 2: Overview of the six exemplar physicalizations used in this study (phys1 - phys6).

between 2D visualization concepts [27] and our observations of reconfiguration in 3D space. To give an example, we discuss how organization in 2D space (i.e. separability and integrality [27]) relates to proximity and atomic orientation changes in 3D space. Following 'data-agnostic' studies on 2D visual perception [3,11,30], we use data-agnostic 'abstract' 3D shapes to study interaction strategies based on proximity, orientation, and tangibility - not a specific dataset. This allows our study into interaction strategies to be independent of any dataset or domain, and more generally informative to future developments in this space. This is further supported by the use of a technology-agnostic setup (effectively accomplished through the use of static cuboids), allowing our findings to inform future technology approaches to physicalizations.

Our methodology, therefore, adopts the apparatus, number of participants and setup from prior work [29], although we fundamentally study a different aspect of interaction with physicalizations. Where Sauvé et al. [29] showed the direct relation between user orientation and perception of static physicalizations that could not be manipulated, this work studies the interactions with those physicalizations. As such we can start to understand how interactivity with physical data, and reconfiguration mechanisms used therein, can be supported in future composite physicalizations.

\subsection{Limitations of the approach}

This methodology enables the distillation of fundamental insights into basic interactions with physicalizations, but does not provide an exhaustive list of potential interactions with diverse physicalizations. Rather, this approach isolates one aspect of the physicalization (i.e. size [18], orientation [29], or in this paper: spatial mapping) to provide evidence on how these isolated characteristics of physicalizations influence users. We focus on a subset of physicalizations, physical bar charts, as these are well-established in the field (i.e. [9, 24, 34]). However, we can not make conclusive statements on the reconfiguration of other types of physicalizations.

\section{METHOD}

The overarching goal of this study is to investigate data reconfiguration strategies on exemplar physicalizations. Specifically, we examine reconfiguration in physical bar-chart style composite physicalizations.

In this work, we use data reconfiguration to refer to the manual rearrangement of physical data objects to (re)organize a physicalization. This study adopts the apparatus, number of participants and setup from [29], and seeks to answer the question: how do users reconfigure exemplar composite physicalizations with two different levels of restriction? This question allows us to examine the intertwined relation between user actions, physicalization structure and reconfiguration characteristics.

The two levels of interaction phases (two levels of restriction) were introduced to examine the extent to which participants can reorganize physical data objects and whether they use different reconfiguration strategies. Our study setup focuses solely on interaction, giving participants a degree of freedom in reorganizing data points that in 'real physicalizations' would break the data consistency. However, this approach enables us to reveal more general reconfiguration strategies that are not specific to one data context.

\subsection{Physicalization design}

The design of the six exemplar physicalizations (referred to as phys1 - phys6; see Figure 2) is informed by the well-known physical bar charts previously used in physicalizations $[5,34]$. This set of physicalizations intentionally contains edge cases of different visual encoding. We thereby ensured that these included various visual obstructions, gradual and abrupt height differences, as well as clear and ambiguous distinctions of clusters, see Figure 2. This allows us to study reconfiguration strategies across a variety of spatial mappings, thoroughly exploring their effect on physical interaction with these mappings. Similar to work on 2D visualization [27], we varied the size (volume) and position (proximity), where we included the rotation of data objects in physical space. Each of the 
physicalizations consists of 16 blue acrylic objects that have four different lengths (Figure 3). This allowed us to create physicalizations with a range of complexity, while maintaining a similar density.

The layouts of the six physicalizations are not based on a concrete dataset, but on intrinsic and relational properties of objects, following the standard definition of physicalizations [17]. Our stimuli follow a set of rules based on three pre-attentive visual properties known from 2D visualization [36]: proximity, continuity, and atomic orientation. We use proximity to differentiate between internal and external distances between clusters of objects on the 2D plane and created two types of spatial relations: in a grid (e.g. Figure 2, phys1) or a linear manner (e.g. Figure 2, phys3). We use continuity to differentiate between perceived connectedness by using objects of similar size or objects of increasing size consecutively. We use atomic orientation - which is the rotation of an object around its own axes - to differentiate in the orientation of single objects within the physicalization, in the $\mathrm{x}, \mathrm{y}$, or $\mathrm{z}$ plane.

\subsection{Setup}

The lab-based study setup consisted of a white fixed square canvas $(40 \times 40 \mathrm{~cm})$ and a camera above the table captured a top-down view of the participants' reconfigurations (Figure 3). We presented each of the six physicalizations from four sides to reduce the previously found influence of orientation [29], creating a total of 24 trials, which were randomized using the Latin square method.

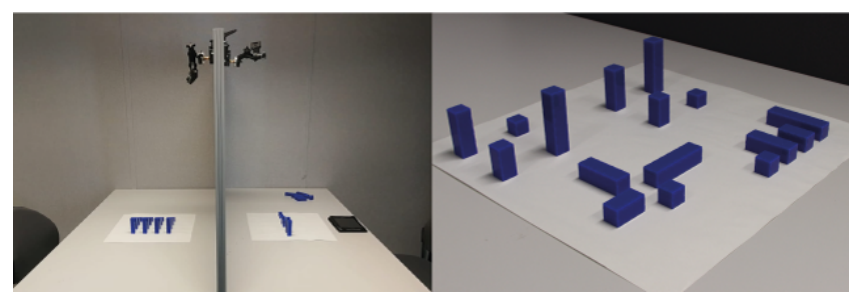

Figure 3: Study setup and exemplar physicalization made with acrylic objects.

\subsection{Participants}

A total of 20 participants were recruited (9 identified as female, 11 as male) with an average age of 27 years $(\sigma=5.92)$. The requirements for participation were that participants are fully (or corrected to fully) sighted and are physically able to reorganize physical objects.

\subsection{Procedure}

We introduced the participants to the study, asked them to sign a consent form and collected their demographics. We explained the goal of the experiment - to understand how people reconfigure physical objects that represent (abstract) data - and walked them through an example trial to familiarize them with the procedure. We did not constrain participants in their reconfigurations, apart from that they were not allowed to stack the individual objects, as this would potentially alter the shape of the data points, rather than the presentation mapping on which this work focuses. We applied a think-aloud method for which we motivated the participants to speak their thoughts aloud while completing the trials as described below. During the study, we randomly presented participants with the six physicalizations, each seen from four sides, creating a total of 24 different physicalizations.

\subsection{Tasks}

The tasks are based on the process of presentation mapping [37], focusing only on the spatial organization of data. We asked participants to reorganize the physicalizations according to the clusters they perceived, making use of the canvas and available physical objects as constraints. The design of the tasks was influenced by Kirsh [20] who proposed that an effective means to simplify the perception of physical (data) objects is to spatially arrange them to reflect one's representation of the task. We deliberately use two levels of restriction (we refer to them as phases) to represent different 'degrees of freedom' found in related work: the change of a single object (phase 1 - restricted) relates to interaction of limited form in interactive systems $[9,34]$ and no restrictions (phase $2-$ unrestricted) relates to complete freedom of interaction in work on constructive visualization $[14,37]$.

\subsection{Participant instructions}

Specifically, we asked participants, if they thought it was possible, to make the clusters they identified more distinct by reorganization in two phases. To make the concept of 'data clustering' more accessible, we used the terminology 'identifying groups', and explained the definition of a group as a set of objects that you think belong together; it is not about the atomic properties of each object, but about their relation to each other. For each of the 24 physicalizations, the following two exact questions were sequentially asked:

Phase 1 (restricted): How would you make the groups you identified more distinct by moving one object? To capture how the participants would reorganize the observed clusters with restriction, we asked them how they would make the clusters more distinct if they could only change one object. We asked them to perform the reconfiguration to capture the exact changes in the object's location and orientation on the canvas.

Phase 2 (unrestricted): How would you make the groups you identified more distinct by moving the least number of objects? To capture how the participants would reorganize the observed clusters with no restriction, we asked them to continue on their result of phase 1 and perform another reconfiguration to capture the exact changes in objects' location and orientation on the canvas.

\subsection{Data collection \& analysis}

We used worksheets with visual representations of the physicalization to capture the answers of the participants to the questions, and we annotated the changes made to the physicalization. To avoid ambiguity in capturing the changes the researcher would ask for clarifications in case user interactions were not explicit.

We made top-down video recordings of the tabletop and the hands of the participants, and audio-recorded their verbal feedback. We particularly considered participants' quotes to verify their intentions and to allow for more detailed reasoning. Lastly, the worksheets that the researcher used during the experiment were cross-referenced with the video recordings of the whole interaction. 
We created a visual library to capture the end results of the changes made by each participant, for phase 1 and phase 2 . These were visual representations of the changes made, to capture the high-fidelity information of the physical manipulations performed on the physicalizations.

To analyze the reconfiguration strategies in more detail, we coded each phase, recording each object's position and orientation. This allowed us to cross-reference coordinate and atomic orientation changes of objects with the clustering data from prior work [29] to extract more detailed information in relation to the clusters identified. Hence, we can discuss change in cohesion, which refers to the distance of objects to the centroid (mean coordinate) within a cluster, and change in separation, which refers to the distance between centroids of clusters of objects. The calculation for cohesion and separation corresponds with common internal evaluation methods for cluster analysis. More specifically, we follow the approach used in the Davies-Bouldin index [4], in which they refer to intra- and inter-cluster similarity - our cohesion and separation respectively. The cluster validation is based on a $2 \mathrm{D}$ plane, not 3D space (as participants were not allowed to stack objects). The performed reconfigurations in phase 1 are inherently included in the result at phase 2 due to the sequential nature of these phases. As such, the results of phase 2 represent all changes performed per trial.

\section{FINDINGS}

Across all participants and physicalizations, for both the restricted and unrestricted conditions, we observed a diverse range of interaction approaches. Our findings categorize these into two predominant reconfiguration strategies: (i) change in proximity and (ii) change in atomic orientation. Overall, our study shows that regardless of restrictions - participants were able and comfortable to perform reconfigurations to make the perceived clusters more distinct. In this section, we describe the observed reconfiguration strategies and how they can inform future work on interactions with dynamic composite physicalizations. We report on (i) the general reconfiguration strategies, (ii) the reconfiguration characteristics per physicalization, and (iii) discuss each of the reconfiguration strategies in further detail. We evidence our observations with descriptive statistics and discuss a selection of concrete examples from the six exemplar physicalizations.

\subsection{Overall reconfiguration strategies}

We present an overview of the general reconfiguration strategies used for the reorganization of physical data objects across all physicalizations and participants. Overall, we observed changes in proximity and atomic orientation as the two main reconfiguration strategies. Of all (480) trials, $52 \%$ involved the reconfiguration of an object at phase 1 (250 trials). These reconfigurations all involved proximity changes of which almost a third additional atomic orientation changes (and 1 trial contained a removal strategy). This means that for $48 \%$ of all trials no reconfiguration was performed at phase 1 . Of all (480) trials, $80 \%$ involved the reconfiguration of one or more objects at phase 2 (386 trials). These reconfigurations all involved proximity changes of which almost a fourth additional atomic orientation changes (and 19 trials contained other strategies). This means that for $20 \%$ of all trials no reconfiguration was performed at phase 2 .

To conclude, when only a single object could be reconfigured (phase 1), participants did so for approximately half of all trials (52\%). When multiple objects could be reconfigured (phase 2), participants did so for the majority of all trials (80\%). This means that with no restrictions at all, participants were most likely to make changes to the exemplar physicalizations. For both phase 1 and 2, all reconfigurations involved the change of proximity, and a minority (respectively $14 \%$ and $16 \%$ of all trials) involved the change of atomic orientation. So, regardless of restrictions, and if an action was taken to make clusters more distinct, changes in the proximity of objects were always used, sometimes (14-16\%) in combination with the rotation of objects.

\subsection{Reconfiguration characteristics per physicalization}

In this section, we elaborate on the reconfiguration characteristics across phases per physicalization. To reiterate, for each physicalization there exists a total of 80 trials ( 20 participants $\times 4$ orientations), each of which involves phase 1 (restriction of changing one object) and a continuation into phase 2 (no restrictions). Table 1 column 3 shows the number (and percentage) of trials that involved the change of a single object (phase 1) and the change of multiple objects (phase 2). The subsequent columns of Table 1 provide numbers of the different reconfiguration strategies within each phase.

5.2.1 Terminology. To describe the reconfiguration characteristics observed in our study, we introduce and define the following terminology for changes in the physicalizations after interaction:

C1 Proximity Change: one or more objects are relocated in the same plane. For example, all objects in phys 1 are moved closer together (see Figure 1, left). Relocation is calculated based on each object's coordinates at their center on the $\mathrm{x}$ and y plane.

C2 Cohesion Change: objects have changed proximity (C1) such that the average distance of all the cluster's objects to the cluster's centroid has changed. For example, in Figure 1 (left) all objects are moved closer together, increasing the cohesion of their clusters, but leaving the separation of clusters (C3) unchanged.

C3 Separation Change: objects have changed proximity (C1) such that the average distance between all clusters' centroids has changed. For example, in Figure 1 (middle) all objects are further apart, increasing the separation between clusters, but leaving the cohesion within clusters (C2) unchanged.

C4 Atomic Orientation Change: one or more objects are rotated over any of their axes. We categorize three different atomic orientation changes we observed in our study: (i) rotation within the plane (x $\leftrightarrow \mathrm{y}$; Figure 12B), (ii) rotation from plane to space $(\mathrm{x} / \mathrm{y} \rightarrow \mathrm{z}$; Figure $12 \mathrm{~A})$, and (iii) rotation from space to plane $(\mathrm{z} \rightarrow \mathrm{x} / \mathrm{y}$; Figure $13 \mathrm{~B})$. For example, a long object laying flat is rotated from pointing east to north (i); is set up straight to point upwards (ii); or was standing and now laid flat (iii). 
Table 1: Occurrence of changes and strategies applied per trial, per physicalization (P) after each phase (ø) across all participants. Cohesion refers to a change in distance of objects within a cluster, and separation refers to a change in distance between clusters. Increase and decrease are indicated by ' + ' and '-' respectively. Percentages per row are in relation to 80 trials per physicalization, the 'Total' row refers to all 480 trials.

\begin{tabular}{|c|c|c|c|c|c|c|c|c|c|c|c|}
\hline \multirow[b]{3}{*}{$\mathrm{P}$} & \multirow[b]{3}{*}{$\varnothing$} & \multirow[b]{3}{*}{ Changes } & \multicolumn{4}{|c|}{ Proximity } & \multicolumn{3}{|c|}{ Atomic Orientation } & \multicolumn{2}{|c|}{ Other } \\
\hline & & & \multicolumn{2}{|c|}{ Cohesion } & \multicolumn{2}{|c|}{ Separation } & \multirow{2}{*}{$\mathrm{x} \leftrightarrow \mathrm{y}$} & \multirow{2}{*}{$\mathrm{xy} \rightarrow \mathrm{z}$} & \multirow{2}{*}{$z \rightarrow x y$} & \multirow{2}{*}{ Swap } & \multirow{2}{*}{ Rem. } \\
\hline & & & + & - & + & - & & & & & \\
\hline \multirow{2}{*}{1} & 1 & $3(4 \%)$ & $2(3 \%)$ & $1(1 \%)$ & $3(4 \%)$ & - & - & - & - & - & - \\
\hline & 2 & $35(44 \%)$ & $23(29 \%)$ & $4(5 \%)$ & $16(20 \%)$ & - & - & - & - & - & - \\
\hline \multirow{2}{*}{2} & 1 & $10(13 \%)$ & $3(4 \%)$ & $5(6 \%)$ & $9(11 \%)$ & - & - & - & - & - & $1(1 \%)$ \\
\hline & 2 & $52(65 \%)$ & $25(31 \%)$ & $10(13 \%)$ & 40 (50\%) & - & - & - & - & $6(8 \%)$ & - \\
\hline \multirow{2}{*}{3} & 1 & $76(95 \%)$ & $56(70 \%)$ & $19(24 \%)$ & $67(84 \%)$ & $44(55 \%)$ & - & - & - & - & - \\
\hline & 2 & 79 (99\%) & $67(84 \%)$ & $23(29 \%)$ & $70(88 \%)$ & $47(59 \%)$ & - & - & $1(1 \%)$ & - & - \\
\hline \multirow{2}{*}{4} & 1 & $74(93 \%)$ & $71(89 \%)$ & $5(6 \%)$ & $68(85 \%)$ & $21(26 \%)$ & $9(11 \%)$ & $37(46 \%)$ & - & - & - \\
\hline & 2 & $80(100 \%)$ & $77(96 \%)$ & $14(18 \%)$ & $72(90 \%)$ & $45(56 \%)$ & $47(59 \%)$ & $46(58 \%)$ & $2(3 \%)$ & - & - \\
\hline \multirow{2}{*}{5} & 1 & $59(74 \%)$ & $14(18 \%)$ & $6(8 \%)$ & $57(71 \%)$ & $2(3 \%)$ & - & - & - & - & - \\
\hline & 2 & $79(99 \%)$ & $34(43 \%)$ & $9(11 \%)$ & $68(85 \%)$ & $12(15 \%)$ & - & - & - & $8(10 \%)$ & $1(1 \%)$ \\
\hline \multirow{2}{*}{6} & 1 & $28(35 \%)$ & $20(25 \%)$ & $4(5 \%)$ & $25(31 \%)$ & $8(10 \%)$ & $14(18 \%)$ & - & $5(6 \%)$ & - & - \\
\hline & 2 & $61(76 \%)$ & $40(50 \%)$ & $10(13 \%)$ & 47 (59\%) & $18(23 \%)$ & $15(19 \%)$ & $1(1 \%)$ & $5(6 \%)$ & $4(5 \%)$ & . \\
\hline \multirow{2}{*}{ Total } & 1 & $250(52 \%)$ & $166(35 \%)$ & $40(8 \%)$ & $229(48 \%)$ & $75(16 \%)$ & $23(5 \%)$ & $37(8 \%)$ & $5(1 \%)$ & - & $1(<1 \%)$ \\
\hline & 2 & $386(80 \%)$ & $266(55 \%)$ & $69(15 \%)$ & $312(65 \%)$ & $122(25 \%)$ & $62(13 \%)$ & 47 (10\%) & $8(2 \%)$ & $18(4 \%)$ & $1(<1 \%)$ \\
\hline
\end{tabular}

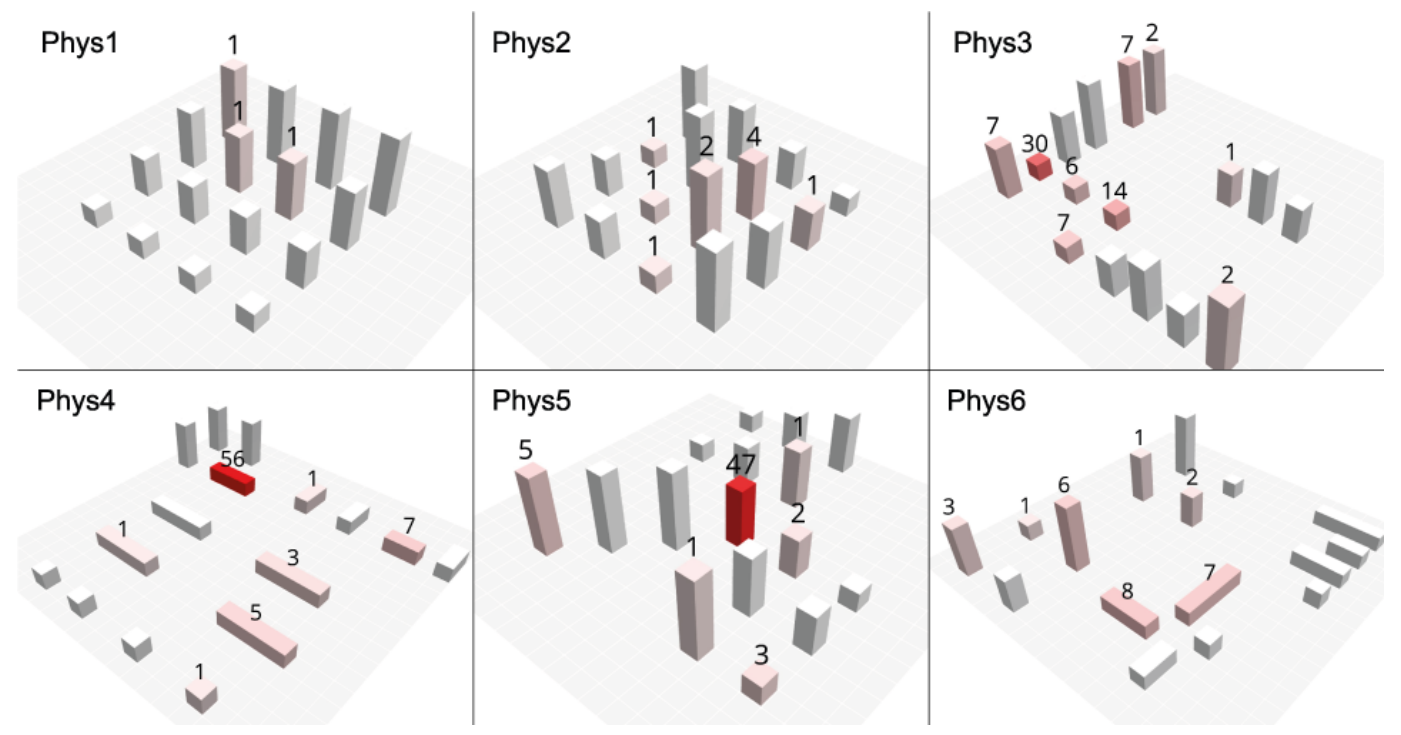

Figure 4: Objects changed at phase 1 per physicalization, ranging from 0 to 56 . Color and annotations show frequency per object ( 0 values are omitted).

5.2.2 Phase 1 per physicalization (restriction of changing one object). Phase 1 represents the limited degrees-of-freedom interaction found in related work on interactive systems (e.g., [9, 24, 33]). Across all reconfigurations at phase 1 (Table 1 ), for phys 1 and phys 2 the majority of trials did not involve the change of a single object. In contrast, for phys3-5 the majority of trials $(95 \%, 93 \%$ and $74 \%)$ did involve the change of a single object. For phys6, 35\% of trials involved the change of a single object.

Figure 4 details the total objects that were changed at phase 1, showing an overview of the accumulated objects that were chosen for each physicalization. For phys6 there was a clear preference for three out of 16 objects (Figure 4, phys6, changed 6-8 times) and for phys3-5 there was a clear preference for a specific single object (Figure 4, phys3-5, changed 30,56, and 47 times respectively).

Considering reconfiguration strategies per physicalization, we observe (see Table 1) that for phys1, phys2, phys3, and phys5 principally proximity changes were made (apart from a minor outlier) and for phys 4 and phys 6 a combination of proximity and atomic orientation changes were performed. 


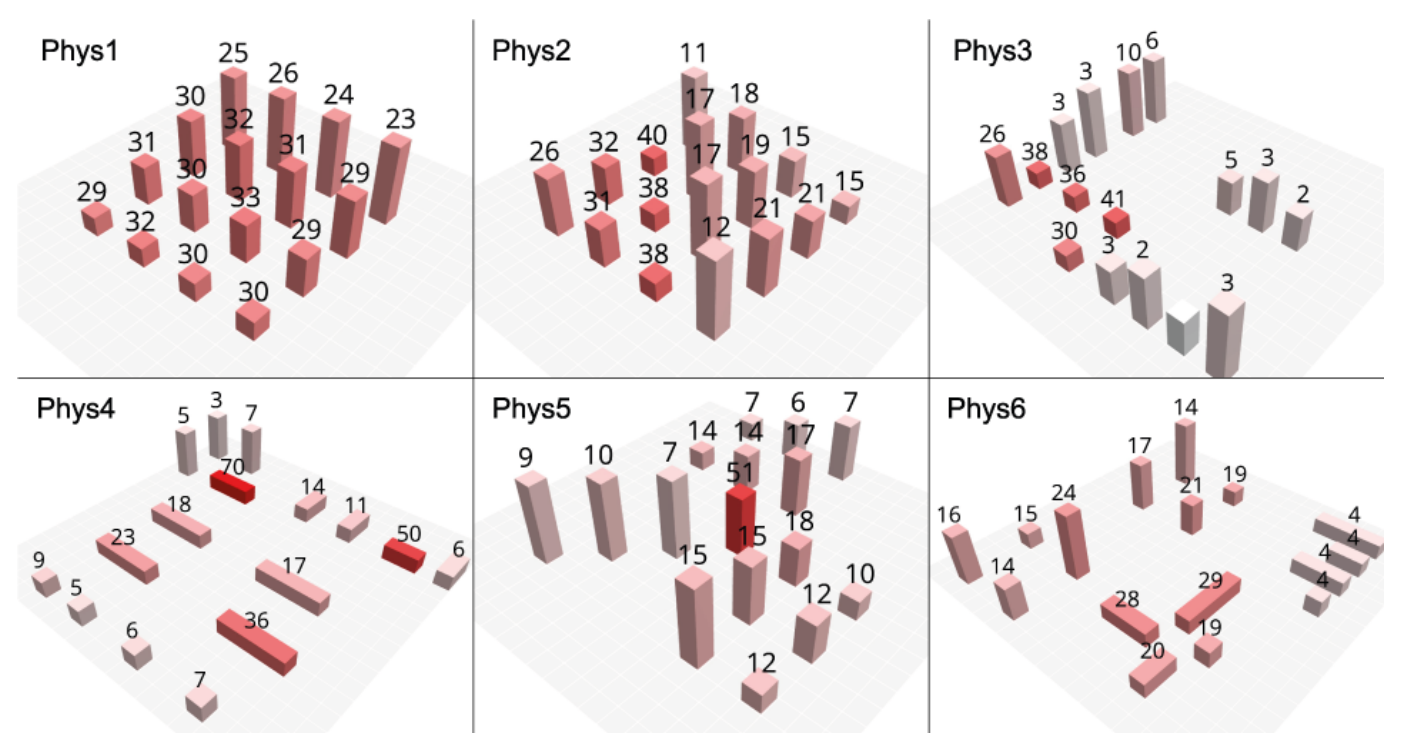

Figure 5: Objects changed at phase 2 per physicalization, ranging from 0 to 70 . Color and annotations show frequency per object (0 values are omitted).

Table 2: The occurrence of changes at phase 2 (column 2) and the frequency of specific amounts of objects changed per trial (in \% of all changes that occurred in that physicalization).

\begin{tabular}{ccrrrrrrrrrrrrrr}
\hline PHYS & Phase 2 & 1 & 2 & 3 & 4 & 5 & 6 & 7 & 8 & 9 & 10 & 12 & 13 & 14 & 16 \\
\hline 1 & 35 & $0 \%$ & $0 \%$ & $0 \%$ & $11 \%$ & $0 \%$ & $0 \%$ & $0 \%$ & $0 \%$ & $0 \%$ & $0 \%$ & $34 \%$ & $0 \%$ & $0 \%$ & $54 \%$ \\
2 & 52 & $4 \%$ & $8 \%$ & $10 \%$ & $13 \%$ & $10 \%$ & $25 \%$ & $2 \%$ & $0 \%$ & $0 \%$ & $6 \%$ & $6 \%$ & $0 \%$ & $2 \%$ & $15 \%$ \\
3 & 79 & $13 \%$ & $43 \%$ & $27 \%$ & $6 \%$ & $6 \%$ & $4 \%$ & $1 \%$ & $0 \%$ & $0 \%$ & $0 \%$ & $0 \%$ & $0 \%$ & $0 \%$ & $0 \%$ \\
4 & 80 & $1 \%$ & $34 \%$ & $26 \%$ & $13 \%$ & $16 \%$ & $4 \%$ & $4 \%$ & $0 \%$ & $1 \%$ & $0 \%$ & $0 \%$ & $0 \%$ & $0 \%$ & $1 \%$ \\
5 & 79 & $35 \%$ & $24 \%$ & $16 \%$ & $10 \%$ & $6 \%$ & $1 \%$ & $1 \%$ & $0 \%$ & $0 \%$ & $0 \%$ & $0 \%$ & $4 \%$ & $0 \%$ & $0 \%$ \\
6 & 61 & $21 \%$ & $11 \%$ & $11 \%$ & $33 \%$ & $3 \%$ & $2 \%$ & $0 \%$ & $11 \%$ & $0 \%$ & $5 \%$ & $5 \%$ & $0 \%$ & $0 \%$ & $2 \%$ \\
\hline
\end{tabular}

5.2.3 Phase 2 per physicalization (continuation of phase 1 with no restrictions). Phase 2 refers to the free-form multiple degrees-offreedom interaction possibilities as seen in related work on constructive physicalizations [13]. Looking at the reconfigurations performed at phase 2 (Table 1) for phys1, less than half of the trials (44\%) involved any change of objects. For phys2 and phys6, this was for more than half of the trials ( $65 \%$ and $76 \%$ ), and for phys3, phys 4 and phys 5 the majority of trials (from $99 \%$ to $100 \%$ ). Figure 5 details the objects that were changed at phase 2, showing an overview of the accumulated objects chosen for each physicalization. Additionally, Table 2 shows the percentages for the total number of objects changed per physicalization.

Combining Figure 5 with Table 2, we can make the following conclusions for each physicalization. Our data shows that for phys1, the majority of reconfigurations involved exactly 12 or 16 objects (34\% and 54\%), with no specific preference for any set of cubes (see Figure 5). In contrast, participants used a varying number of objects for phys2 (Table 2) and showed a slight preference for which objects to reconfigure, reflected in various objects being chosen between 11 and 40 times (Figure 5). Phys6 involved the change of predominantly 1 or 4 objects ( $21 \%$ and $33 \%$ ), which, with a slight preference for the bottom cluster, is reflected by the varying highlighted objects, being chosen between 4 to 29 times. For phys 3 it varied between 1 to 3 objects (13-43\%), with a clear choice of particular objects being changed 36, 38, or 41 times. Similarly, reconfigurations for phys4 primarily involved between 2 to 5 objects (13-34\%), again, with a visible preference for some objects resulting in objects being chosen between 17 and 70 times. Lastly, for phys5, reconfigurations leaned towards 1 to 3 objects (16-35\%), with an overwhelming preference for one object being changed 51 times.

Considering the strategies used per physicalization at phase 2, we observed (see Table 1) that for phys1, phys2, phys3, and phys5 mainly proximity changes dominated (apart from the minor strategy of swapping for phys2 and phys5). For phys4 and phys6 we observed a combination of proximity and atomic orientation changes.

5.2.4 Reconfiguration strategies per physicalization. Combining the object frequency information from Figure 4 and Figure 5, and the occurrence numbers from Table 1 and 2, we can derive a general strategy per physicalization for phase 1 and phase 2. Figure 6 shows that for the majority of physicalizations an increase in cohesion and separation occur simultaneously, although with a different number of objects. Phys1 presents a special case as only cohesion 


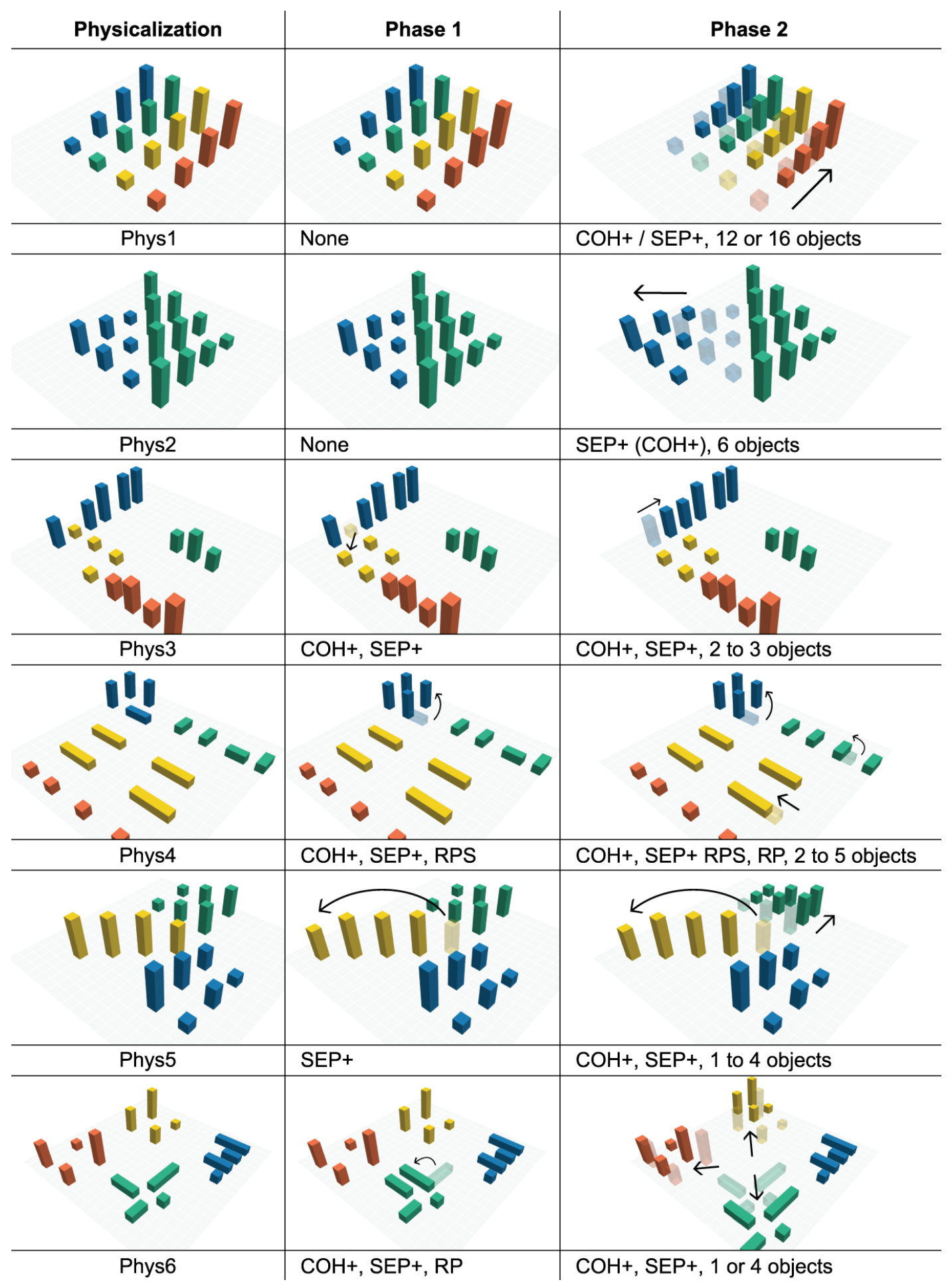

Figure 6: An overview of the main reconfiguration strategies observed per physicalization for both phases, including cohesion increase $(\mathrm{COH}+)$, separation increase $(\mathrm{SEP}+)$, rotation from plane to space (RPS) and rotation in the plane (RP). Colors are used to indicate identified clusters and shadows are used to show object positions prior to the changes made. 
or separation was increased, using 12 or 16 objects (Figure 6, phys 1 - phase 2). Phys2 and phys5 are the only two physicalizations that show an example of an isolated increase of separation (Figure 6, phys 2 - phase 2, phys 5 - phase 1). Lastly, phys4 and phys6 are the two physicalizations in which atomic orientation was part of the main strategy (Figure 6, phys4 - phase 1 and 2, phys6 - phase 1).

For phys 1 and phys 2 the overarching strategy at phase 1 was to not change any objects. The lack of change for these two physicalizations could be explained by the orderly arrangement of all objects, creating a constant internal structure. Therefore, it is difficult to effectively reorganize them by only changing a single object. For phys 3 and phys 5 the main strategy involved the change of a centrally positioned object (Figure 6, phys 5 - phase 1) or one out of a selection of objects that was located on the conjunction of clusters (Figure 6, phys3 - phase 1). Hence, changing object locations was used to 'untangle' the overlapping relations, and improve the organization of the physicalization.

For phys4 the main strategy involved the change of atomic orientation of one or more objects. This was done to visually integrate with the atomic orientation of neighboring objects, while differentiating from the objects lying flat in the plane. This shows that manipulating the atomic orientation change of an object, inevitably influences its cluster's cohesion and separation, as the coordinates of the center of the object change. In this case, (the center of) the object got closer to its cluster centroid (Figure 6, phys4 - phase 1). The mixed orientations of objects within clusters invited participants to make changes to individual objects of different clusters. Lastly, the main strategy for phys6 was similar to phys4. However, at phase 2 we observed the increase of separation and cohesion over atomic orientation changes (Figure 6, phys6 - phase 2).

To summarize, our data shows that exemplar physicalizations with a constant internal structure are likely to involve either none or many changes (see phys1 and phys2); physicalizations representing overlapping cluster borders are likely to motivate a change of one or more centrally positioned objects (see phys3 and phys5) and; physicalizations with objects in mixed orientations likely result in rotation changes to integrate and/or differentiate their atomic orientation of objects (see phys4 and phys6). This demonstrates that when physicalizations show strong adherence to pre-attentive visual properties, then few if any interactions are performed - and vice versa. This means that clusters that are visually separate and have consistent orientations within, do not motivate participants to reconfigure them. Conversely, ambiguous separation and orientation of clusters result in reconfigurations to reduce that ambiguity.

\subsection{Reconfiguration strategies across all physicalizations}

In the following sections, we discuss reconfiguration strategies more generally and in relation to participant actions and physicalization structure. As a reminder, changes in proximity and atomic orientation were most frequently used across physicalizations. Proximity changes occurred for all physicalizations, whereas atomic orientation changes occurred most prominent for phys 4 and phys6. In the remainder, we further dissect these two main strategies and report on some outlying strategies that we observed.

\subsection{Proximity as reconfiguration strategy}

In general, proximity changes were observed in 249 trials at phase $1(52 \%)$ and 385 trials at phase $2(80 \%)$. To illustrate, for phys1, we primarily observed either the increase of cohesion, in which participants decreased the distance within clusters (Figure 1 left), or the increase of separation, in which participants increased the distance between clusters (Figure 1 middle). Within our study we observed different ways in which cohesion and separation changes can coexist (Table 1) and we will elaborate on three of these for both phases.

5.4.1 Simultaneous cohesion \& separation changes. The most common proximity approach we observed was the simultaneous increase of cohesion and separation. For all trials, this strategy was applied 157 times at phase $1(33 \%)$ and 219 times at phase $2(46 \%)$, especially for phys3 and phys4.

To illustrate this behavior for phase 1, we take phys3 as a concrete example, for which behavior of this type was observed across 52 trials ( $65 \%$ of phys 3 trials). As shown in Figure 6 (phys 3 - phase 1 ), the cube that created overlap with another cluster is moved out of that cluster and closer to the objects with similar properties, increasing the cohesion within the cluster and increasing the separation between clusters. For phase 2, similar behavior was observed across 60 trials (75\% of phys 3 trials). Subsequent to phase 1 , an additional object was changed to again increase its cohesion within and separation between clusters (see Figure 6, phys3 - phase 2).

In summary, from all proximity changes observed, the simultaneous increase of cohesion and separation was most common. It can be expected that moving objects closer within a cluster to make them more cohesive, simultaneously also creates more distance between clusters that make them appear more separate. This coupling is expected, since cohesion and separation have an intrinsic relation in our Gestalt-based layouts. We observed that participants applied this strategy as an effective way to create visual consistency and avoid any ambiguity, thus, clarifying the spatial relations of objects.

It remains an open question as to what extent the simultaneous increase of cohesion and separation is intended and/or conscious. For example, from participants' comments it appears that, at times, the focus was mainly on one (increasing separation or cohesion), whilst the other was a 'by-product' of the changes made. Further research would be needed to unravel the sensemaking of users in these reconfigurations, and whether their intentions and resulting layouts are consciously coupled.

5.4.2 Cohesion changes. Looking at cohesion changes in isolation (Table 3, columns 3-5), we distinguish between occasions in which both the increase and decrease of cohesion occurred ( $\mathrm{COH}+-)$ and occasions in which this occurred in isolation from each other (either $\mathrm{COH}+$ or $\mathrm{COH}-$ ). From these three combinations, the sole increase of cohesion was observed most frequently (Table 3, column 3), which refers to shortening the distance of an object or objects to their cluster centroid. For all trials, this approach was taken 156 times at phase $1(33 \%)$ and 233 times at phase $2(49 \%)$.

At phase 1, increasing cohesion often involved the 'pushing in' of an object into the cluster it was assigned to (Figure 7A). At phase 2 , increasing cohesion would be performed by moving multiple objects of a cluster closer together evenly (Figure 7B). 
Table 3: Percentages of the different combinations within cohesion ( $\mathrm{COH}$ ) and separation (SEP) changes across phase 1 and 2 , either for increase only (+), decrease only (-) or both increase and decrease (+-).

\begin{tabular}{ccccccccc}
\hline & & \multicolumn{3}{c}{$\mathrm{COH}$} & & \multicolumn{3}{c}{ SEP } \\
\cline { 3 - 4 } \cline { 7 - 8 } Phase & changes & + & - & +- & & + & - & +- \\
\hline 1 & 250 & $33 \%$ & $6 \%$ & $2 \%$ & & $36 \%$ & $4 \%$ & $12 \%$ \\
2 & 386 & $49 \%$ & $7 \%$ & $7 \%$ & $45 \%$ & $5 \%$ & $20 \%$ \\
\hline
\end{tabular}

Interestingly, of all trials at phase 2, $34(10 \%)$ involved a special increase of cohesion, in which the objects were moved towards each other until they were touching, creating an internal distance of zero (Figure 7C). Nine participants showed this kind of action across physicalizations, and specifically for phys1. Even more noticeable, P4 performed this action for 18 of all their 24 trials.

Although decreasing the cohesion is a somewhat counterintuitive strategy for reorganizing clusters, there were some outliers in which this was applied. One example is P15, who explained that they performed a decrease of cohesion for the single cluster they identified in phys2, to increase the visibility of all individual datapoints (Figure 8A). Further, P19 applied simultaneous increase and decrease of cohesion for $75 \%$ of their trials of phys 1 and phys 2 to disrupt any possible other trends and strengthen the clusters they identified (Figure 8B).

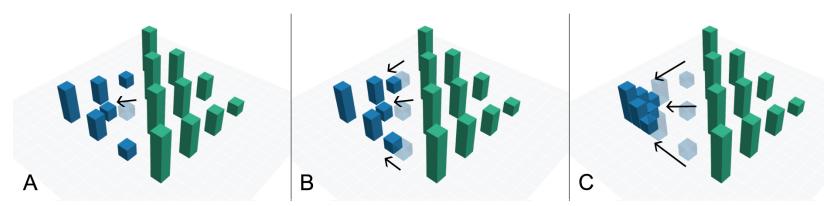

Figure 7: For phys2, an example of (A) phase 1 cohesion increase, (B) phase 2 cohesion increase and (C) a unique approach to cohesion increase until objects are touching.

In summary, looking at cohesion changes in isolation, participants generally performed the increase of cohesion over the other two cohesion behaviors, often by 'pushing in' a single object into their cluster or moving multiple objects together evenly. This behavior resonates with achieving visual consistency within clusters. However, maintaining the exact internal structure of clusters seems to be less important. For example, rather than moving an entire cluster evenly away from another cluster, participants chose to move particular objects closer 'into' a cluster (Figure 7B). This means that the internal structure of the cluster and its location (the centroid) changed as well. In these approaches, the focus is on the cohesion of one particular cluster, over (i) the separation with other clusters and/or (ii) the location of the cluster in the complete canvas.

However, in some cases, deliberate attention to the overall physicalization was observed, resulting in seemingly counterintuitive behavior (i.e. cohesion decrease; Figure $8 \mathrm{~A}$ ) to reduce the occlusion of clusters and data points in the physical space. Here, the layout was treated as a whole, where the focus was on reducing ambiguity
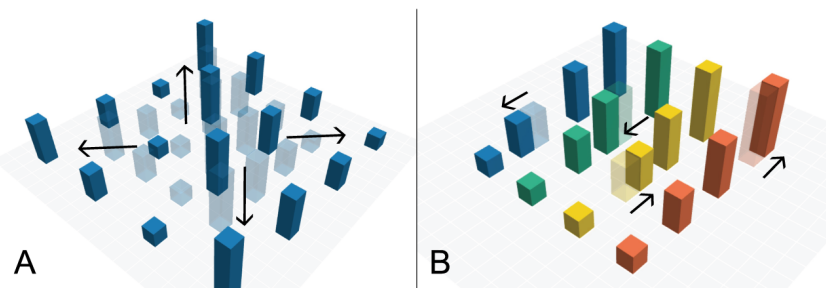

Figure 8: Examples of (A) cohesion decrease for phys2 and (B) simultaneous cohesion decrease and increase for phys 1 .

and/or improving the visual consistency of the entire layout, over the visual consistency and/or ambiguity of a singular cluster.

5.4.3 Separation changes. Looking at separation changes in isolation (Table 3 columns 6-8), we distinguish between occasions in which both the increase and decrease of separation occurred (SEP+-) and occasions in which this occurred in isolation from each other (either SEP+ or SEP-). From these three combinations, the sole increase of separation was observed most frequently (Table 3 column 6), which refers to increasing the distance between the centroids of neighboring clusters. For all trials, this approach was taken 171 times at phase $1(36 \%)$ and 218 times at phase $2(45 \%)$.

The general approach we observed for increasing separation, especially across phase 1 , was the move of a single object to the opposite side of its cluster, to make this cluster appear more distant from the other clusters. A clear example is phys5, as one single object was relocated 47 times at phase $1(59 \%)$. As can be seen from Figure 6 (phys5 phase 1), the relocation of the object does separate the clusters without changing the internal cohesion of that particular cluster (although the order of objects changes).

Across phase 2 we observed a general separation approach of relocating a complete cluster further apart from others, maintaining its internal structure (Figure 9B). However, unique variations of this separation behavior occurred, for example by moving parts of a cluster to increase the separation between (Figure 9C).

In comparison to cohesion, the simultaneous increase and decrease of separation occurred more often across phase $1(12 \%)$ and especially phase $2(20 \%)$. This can be explained by the occasional difference between the verbal explanations of participants and their execution of the change. To illustrate we use phys3 as an example (Figure 10). P8 explained that they wanted to increase separation for the cluster of 3 cubes, which is performed successfully from the largest cluster. However, simultaneously, the cluster of cubes comes closer to the two remaining clusters, decreasing separation.

In summary, looking at separation changes in isolation, participants' actions generally resulted only in an increase of separation, either by relocating a single object to the opposite side of its cluster, or moving a cluster's centroid further away from others. In a similar manner to cohesion, this behavior was expected as it resonates with achieving visual consistency between clusters. However, in contrast to a cluster's internal cohesion, cluster separation involves moving one or more clusters further apart, and, as such, is more dependent on the physicalization canvas as a whole. This potentially explains some observed behavior where participants moved a cluster further away from another, indirectly causing it to come closer to another 


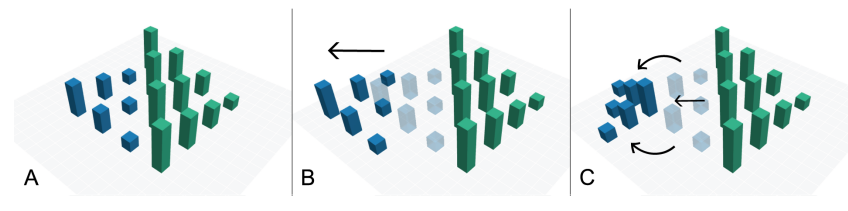

Figure 9: (A) Initial structure of phys2, (B) after separation increase and $(C)$ after separation increase by moving part of a cluster.

A
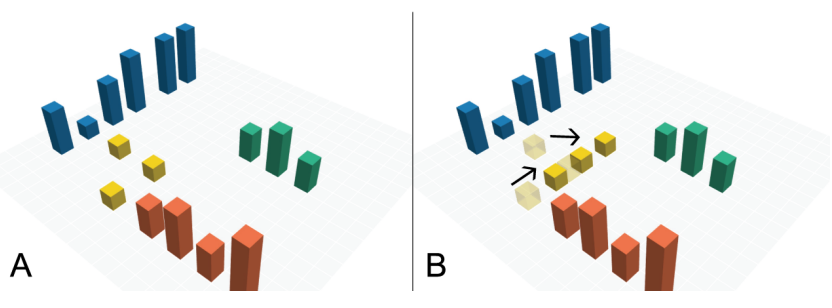

Figure 10: (A) Initial structure of phys3 and (B) after simultaneous separation increase with the largest cluster and decrease with the two remaining clusters.

neighboring cluster. Depending on the participant and task at hand, this might not have been the focus point of the participant.

It is important to note that cluster separation is rather susceptible to internal restructuring, as not only relative internal distances change, but also absolute locations (cluster centroids). To give an example, moving an object to the other side of its cluster, could leave the cohesion unchanged. However, this does alter the centroid's location and therefore the distance to any neighboring clusters. Since a cluster can contain more than one object, the potential to affect separation increases. Given our measure of separation (centroids' distance to their closest neighbors) many unintentional separation changes (either increase or decrease) could occur.

5.4.4 Discussion: Proximity as reconfiguration strategy. Within the reconfiguration strategy of proximity, the overarching approach was to simultaneously increase cohesion and separation. In other words, moving objects of a cluster closer together while also moving them as a cluster further apart from neighboring clusters. We additionally observed both approaches used in isolation (e.g. only cohesion) with a few combinations, some more intuitive than others (e.g. simultaneous increase and decrease of cohesion).

Our observations show parallels with 2D evaluation concepts such as separability and integrality [27], which refer to the extent to which multiple visual channels can be perceived or attended to independently. The simultaneous increase of cohesion and separation results in objects within a cluster becoming more integral, and objects between clusters becoming more separable. Hence, this is an effective means to create visual consistency in a physicalization and reduce any ambiguities.

Our observations also show differences with $2 \mathrm{D}$ visualization, in that perspective plays a central role in the perception of physical distance and space. Concepts such as separability and integrality can be perceived differently in $3 \mathrm{D}$ space compared to $2 \mathrm{D}$ space. For example, moving two clusters further apart so that they are positioned left and right from the viewer (along the x-axis) might visually appear more separate than two clusters being far and close (along the y-axis), as after moving them apart they might still occlude each other visually.

Counterintuitive behaviors can be discussed in light of physical distance and space as well. Those that we observed, such as simultaneous increase and decrease of separation, or the disruption of possible other trends, are difficult to explain from a purely visual encoding point of view. However, when taking into account the possible difference that exists between perceived physical information and actual information, these behaviors can be explained by looking at visual occlusion that can occur in physical space.

The observed simultaneous increase and decrease of separation can be explained by participants not seeing the true proximity between certain (clusters of) objects due to their perspective. In other words, some (clusters of) objects might appear visually closer or further apart than they actually are, depending on the perspective of the viewer, which affects the reconfigurations participants deem important to make the cluster(s) more distinct. This resonates with prior observations of proximity occlusion [29].

Likewise, the observed behavior of deliberately disrupting trends could be explained by participants not seeing the true continuity of certain (clusters of) objects due to their perspective. In other words, some (clusters of) objects might appear visually more or less connected than they actually are, which affects the reconfigurations participants deem important to make the cluster(s) more distinct. This resonates with prior observations of continuity occlusion [29].

\subsection{Atomic orientation as reconfiguration strategy}

Atomic orientation change occurs when one or more objects are rotated in any of their axes. In general, this reconfiguration strategy was performed for 66 trials at phase $1(14 \%)$ and 78 trials at phase 2 $(16 \%)$. Herein, we discuss three different approaches we observed in our study: (i) rotation within the plane, (ii) rotation from plane to space, and (iii) rotation from space to plane. We elaborate on each of these in the following sections, for both phase 1 and 2, and illustrate them by concrete examples from the physicalizations.

5.5.1 Rotation within the plane. We observed the approach of rotation within the plane 23 times at phase $1(5 \%)$ and 62 times at phase $2(13 \%)$, exclusively for phys 4 and phys6. As a concrete example, we discuss the changes made for phys6 at phase 1 (Figure 11). All the observed rotations in the plane were performed using one of the two tallest objects of the green cluster in the bottom left of the plane (Figure 11A). Participants had different explanations for their actions, and performed it to either (i) increase separation to the closest upright cluster (Figure 11B), (ii) integrate with the atomic orientation within the cluster (Figure 11B), or (iii) increase separation to the other flat-lying cluster (Figure 11C). Lastly, at phase 2, two participants reverted their change and performed separation increase instead, preserving the initial structure of the cluster (Figure 11D).

To summarize, rotations within the plane of one or more objects were performed with different intentions, such as increasing separation or differentiating atomic orientation with other clusters. These 


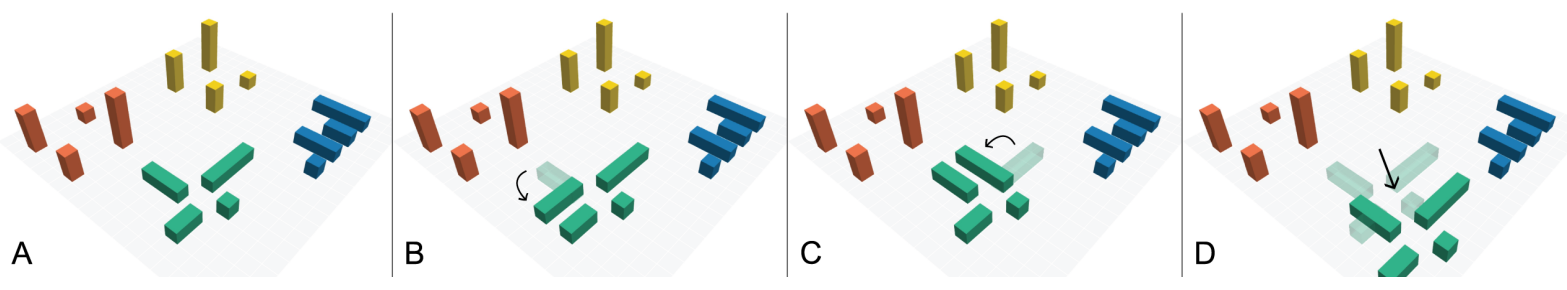

Figure 11: (A) Initial structure of phys6, and after rotation in the plane to either (B) increase separation with the upright cluster, (C) differentiate atomic orientation with the other flat cluster and (D) for phase 2 separation increase instead.

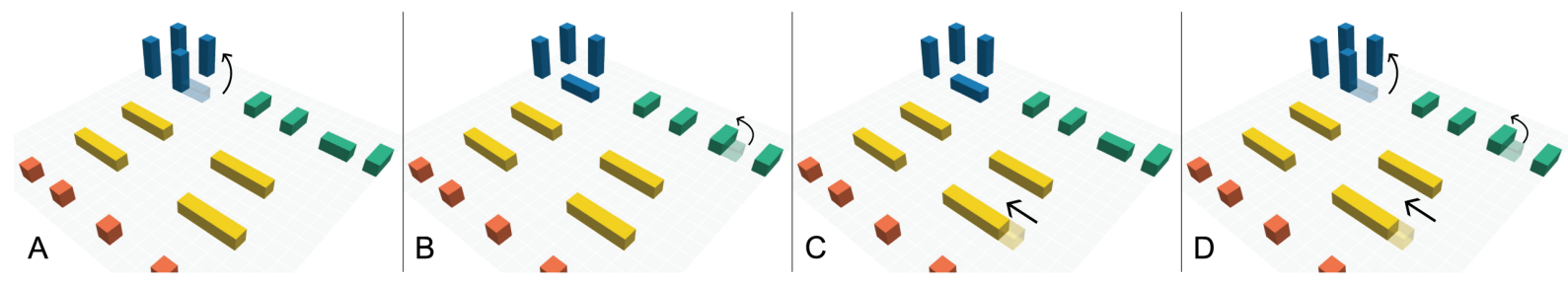

Figure 12: For phys4, (A) phase 1 rotation from plane to space, (B) phase 1 rotation within the plane, (C) phase 1 cohesion increase and (D) although 3 different phase 1 actions, the same result at phase 2.

rotations in the plane exclusively occurred for phys4 and phys6, which can be attributed to their objects having mixed orientations.

All rotations in the plane were performed using one of the larger objects. This is likely because their size has a larger impact on the visual consistency when its orientation changes, compared to smaller objects. The large (i.e. wide or deep) objects look visually different from the viewer's perspective when it is observed from a different angle. Therefore, especially with the restriction of changing only one object, rotating larger objects is an effective means to make clusters more distinct and improve the overall visual consistency.

5.5.2 Rotation from plane to space. We observed the approach of rotation from plane to space 37 times at phase $1(8 \%)$ and 47 times at phase $2(10 \%)$. However, looking solely at phys 4 , rotation from plane to space was performed 37 times at phase $1(46 \%)$ and 46 times at phase $2(58 \%)$. Hence, we will discuss phys 4 as an example to illustrate this approach.

The general approach we observed across trials and phases for phys 4 was the plane to space rotation of a specific flat-lying object (37 times), for it to integrate with the atomic orientation of its neighboring same-sized objects of the same cluster (Figure 12A). In contrast to phase 1 , in which this single rotation prevailed, in phase 2, two different types of rotations were performed equally as often (46-47 times; Figure 12A \& B). So, in addition to the rotation from plane to space of the flat-lying object, a rotation of an object within the plane was performed.

As can be seen from Figure 12, three different approaches observed for phys 4 at phase 1 , would result in a similar final layout at phase 2. From the participant comments, it became clear that these different approaches related to the extent to which they perceived objects of similar size either similar or different due to their atomic orientation. Participants aimed for visual alignment and made the collective atomic orientation more consistent per cluster; in the restricted phase 1 this led to varied rotations as first preference, however, in the unrestricted phase 2 led to a similar visual outcome.

In summary, for rotations from plane to space, we generally observed that rotations of objects were performed to make the objects integrate more with the atomic orientation of the cluster they were assigned to. This held true for single changes (phase 1) and multiple (phase 2).

The layout with mixed orientations in phys4 motivated the approach of rotating objects from plane to space - and in particular a specific flat-lying object in the blue cluster. Due to its stark visual ambiguity within that cluster (i.e. being the 'odd one out'), it was primarily chosen in this approach (37 times). Hence, placing that object from flat to upright proves to be an effective way to improve the visual consistency of that particular cluster - more than a rotation within the plane would do for, i.e., the green cluster. This suggests that not all types of rotations in $3 \mathrm{D}$ space result in equally impactful visual results, and that rotations within the plane (between $\mathrm{x}$ and $\mathrm{y}$ ) might be perceived less impactful than rotations from plane to space (from $x y$ to $\mathrm{z}$ ) or vise versa (from $\mathrm{z}$ to $\mathrm{xy}$ ).

In contrast to rotation within the plane (discussed earlier), rotation from plane to space was not frequently observed for phys6 $(\leq 1 \%)$. We observed that although the layout of phys6 uses mixed orientations, there is a stark separation in the proximity between four potential clusters, and within these clusters the orientation is fairly consistent - especially their orientation towards space. Hence, participants were less inclined to make rotations from plane to space to improve visual consistency.

5.5.3 Rotation from space to plane. We observed the approach of rotation from space to plane 5 to 8 times at phases 1 and 2 (1-2\%), only across 3 physicalizations. As a concrete example, we discuss the changes made by $\mathrm{P} 5$ for phys 6 at phase 1 . We observed that they performed consistently the rotation from space to plane of one of the tallest upright objects (Figure 13). They and one other participant 
that performed this exact behavior (P11) explained that they aimed to differentiate between the two upwards clusters, by decreasing the consistency in object orientation of one of the clusters. In this way they would not have similar consistencies in orientation, hence become more distinct.

For phys 3 at phase 2 we observed a unique case in P1, as they rotated one of the clusters from space to plane and explained that they intended to differentiate between the two clusters by changing the atomic orientation of one complete cluster (Figure 14).

In summary, for the approach of rotation from space to plane, we generally observed that rotations of one or more objects were performed to create a differentiation between clusters by creating inconsistencies in orientation. In phys6, for example, instead of strengthening the visual consistency within the clusters (as was seen for previous two atomic rotation approaches), an ambiguity was introduced to make the cluster differ from another cluster that would otherwise appear similar. Likewise, in phys3, a complete cluster was made more ambiguous, to make the cluster stand out from the rest of the physicalization layout. We conclude that rotation from space to plane is particularly useful to create local ambiguities, to improve the distinction of clusters in the overall layout.

5.5.4 Discussion: Atomic orientation as reconfiguration strategy. Within the reconfiguration strategy of atomic orientation we observed three approaches: (i) rotation within the plane, (ii) rotation from plane to space, and (iii) rotation from space to plane. In general, we observed that rotations within the plane and rotations from space to plane were performed to increase separation and/or differentiate the atomic orientation with other clusters. For rotation from plane to space, the intention was to make the object integrate more with the atomic orientation of the cluster it was assigned to.

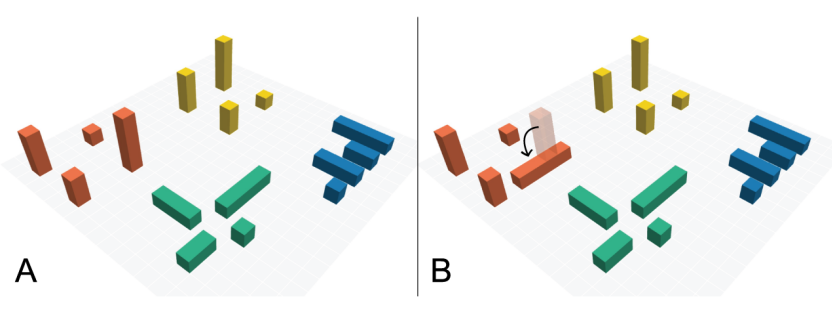

Figure 13: (A) Initial structure of phys6 and (B) after rotation of a single object from plane to space.

A

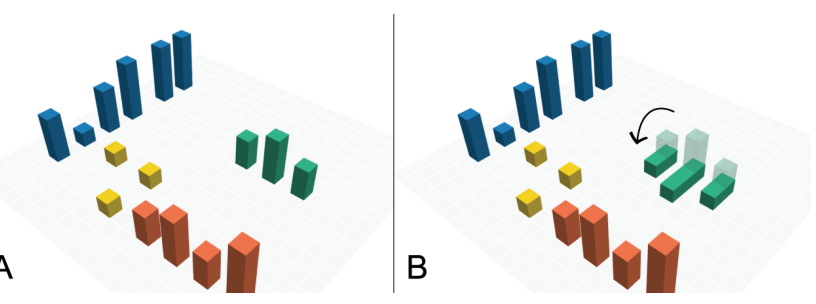

Figure 14: (A) Initial structure of phys3 and (B) after cluster rotation from space to plane.
As opposed to flat 2D visualizations, which allow for rotation in solely $\mathrm{x}$ and $\mathrm{y}$ directions, rotation in physical space with the additional z-axis has different implications. We observed that rotations could either be used to integrate within or differentiate between clusters, which again shows parallels with the notions of integrality and separability from 2D visualization work [27]. However, it remains an open question to what extent rotations across the three axes are perceived similarly or equally, such as rotations in the plane versus rotations from plane to space. One example is, as illustrated in interactions with phys3 (Figure 14), a complete cluster that was reconfigured from space to plane to bring them to a 'different dimension' in relation to the clusters standing upright. Differently, for phys6, the atomic orientation change of a single object within a cluster was used to differentiate between clusters. Hence, it can be questioned if rotations across all three axes are perceived and utilized similarly, or that particular types of rotation can be used for different intentions and can have different implications.

We connect our work to prior findings from psychophysics on the notion of radial-tangential illusion [2], which describes that lengths represented away from and towards the body are perceived to be larger than lengths that are presented from side to side to the user. This indicates that the perceived length of an object depends on its orientation. Since we applied four orientation conditions for each of the six exemplar physicalizations, this would result in objects in some orientations to be perceived as 'tall' whereas in other orientations as 'wide'.

To give another example, if one observes two objects of similar size, one standing upright and one lying flat away from the user, they might be perceived more similarly, then the two same objects but then standing upright and one lying flat from side to side to the user. This can be explained by atomic orientation occlusion [29], which describes that due to different atomic orientations of objects, objects of similar size might look different and objects of different size might look similar.

To conclude, atomic orientation occlusion of physical data objects interacts/interferes with the concepts of integrality and separability in ways different to $2 \mathrm{D}$ visualization. Hence, it invites for further reconfiguration possibilities and/or different layers of information. It could be further explored how we can assign meaning to different types of rotation of data objects in physical space.

\subsection{Other reconfiguration strategies}

We observed other minor reconfiguration strategies for a total of 20 times across phases 1 and 2 (1-4\%) but only for 3 physicalizations. We discuss all changes made, in which no proximity change and/or atomic orientation change occurred. These minor strategies almost exclusively occurred at phase 2 .

The swapping of objects was observed 18 times at phase $2(4 \%)$, across three physicalizations. Swapping refers to the instances in which no proximity or atomic orientation changes have been made, but two or more objects have swapped their location on the canvas. This approach generally was performed to either strengthen the perceived patterns or disrupt them further to put the emphasis on one larger trend. One example is P14, who performed the swapping of objects for $75 \%$ of their trials for phys5 (Figure 15B) to let the trend of that cluster integrate with the other increasing trends. 

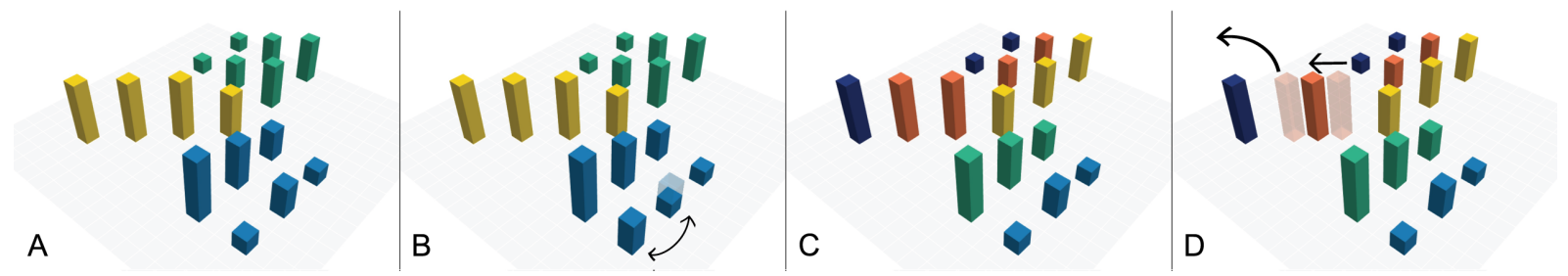

Figure 15: (A) Initial structure of phys5 as perceived by P14 (3 clusters) and (B) after the swapping of 2 objects (bottom left) to increase cohesion. (C) Initial structure of phys5 as perceived by P6 (5 clusters) and (D) after the removal of an object.

Although removal was not part of the procedure instructions, we observed 2 cases in which a single object was removed. This was performed by $\mathrm{P} 15$ at phase 1 for phys2, and by P6 at phase 2 for phys5 (Figure 15). P6 removed one object and aligned the others to make sure all $\mathrm{x}$ and $\mathrm{y}$ rows and columns contained 3 objects. P15 explained they removed the object as they wanted to prevent possible other trends to be seen in the physicalization structure.

To summarize, in 20 trials, the reconfiguration strategies of swapping and removal were used to either strengthen the perceived patterns or to reduce the visibility of other trends. The swapping of objects was thereby used to improve the overall visual consistency of the physicalization layout, where the absolute location of the cluster (the centroid) remained unchanged. Visually, the changes made through swapping appear more subtly, as it improves the overall visual consistency (i.e. trends) without changing the location of a cluster. The removal of objects was used to 'declutter' the layout from any ambiguous data point or ambiguity in the trends perceived. However, whether and how we should anticipate this reconfiguration strategy is uncertain, as users effectively remove data points in the task of making clusters more distinct.

\section{DISCUSSION}

In this paper, we investigated people's spontaneous reconfiguration strategies for the reorganization of exemplar physicalizations (from the physical bar chart archetype) to make clusters more 'distinct'. Our findings show that proximity change was generally the most used strategy to reorganize clusters, primarily resulting in increased cohesion and separation. We observed that participants performed few or no changes in physicalizations with a constant internal structure (such as phys1 and phys2). Likewise, where the physicalization had a less distinct structure and higher separation (such as phys6) no changes were made. If physicalizations adhere strongly to perceptual properties, i.e. they are visually consistent and do not contain ambiguities, there is no need to manipulate them. In contrast, for more complex physicalizations, i.e. with mixed orientations or objects in a diagonal line (such as phys4 and phys5), diverse changes were made. If physicalizations adhere weakly to perceptual properties, i.e. the orientations are visually ambiguous, users are inclined to manipulate them to reduce or eliminate these ambiguities. Overall, our results suggest that future (dynamic) composite physicalizations should consider proximity changes as a main form of interaction, as it proves an effective way of improving the visual consistency and reducing ambiguity in a visualization.

\subsection{Differences between reconfiguration strategies with or without restrictions}

We observed different reconfiguration strategies when comparing the two study phases. In phase 1 (with restriction of changing one object), we generally observed participants increasing cohesion by 'pushing' an object into a cluster, often compromising the initial cluster structure. For phase 2 (no restrictions), increasing cohesion was achieved by moving objects of a cluster closer together. Similarly, when increasing separation in phase 1 participants would move an object to the other side of its cluster, visually changing the initial cluster structure, whereas for phase 2 the complete cluster would be relocated (maintaining the initial cluster structure). In other words, when participants were restricted to changing one object, they would sometimes compromise the visual consistency of the initial cluster structure, to increase cohesion and/or separation, whereas with no restrictions, they choose to maintain the cluster structure. Our study showed that regardless of more or less restrictions, participants were able to reorganize the physicalizations. As the design of composite physicalizations requires the consideration of the level of granularity, degree of manipulability, and level of actuation [23] this poses the question of what combination of limitations and freedom will support users in reconfiguring data. This opens up further questions about the extent to which future composite physicalizations should dictate and/or restrict the intended interaction with data.

\subsection{Proximity and atomic orientation as novel encodings in $3 \mathrm{D}$ space}

Our findings are in line with related work from shape-changing interfaces and constructive visualization that showed that proximity was used to differentiate between clusters of data [13, 34]. For example, in EMERGE [34], participants would hide irrelevant data to either emphasize or create a barrier between grouped rows, and with constructive token visualizations [14], participants would create differences in proximity in the canvas to show which data was more or less related within the visualization. These actions are equivalent to the increase of cohesion and separation we observed in our study, and also show parallels with the concepts of integrality and separability of 2D visualization [27]. However, related work remains close to the traditional visualization concepts of columns and rows, whereas the physical representation of data invites the more free-form use of 3D space. While work on constructive visualization [13] showed that some participants freely made use of the 3D space (e.g., creating row and columns, but also combinations of 
plane and stacked organizations), actual interactive systems such as EMERGE [34] and ShapeCanvas [7] provide very limited physical manipulations considering data points are fixed and can only be moved up or down.

Extending related work, our work illustrates that participants were confident in performing spatial data object organizations on abstract physicalizations that went beyond the traditional representation of data. Although proximity changes came out as most effective to reorganize physical data objects, our study also opened up the consideration of atomic orientation as a way to reconfigure data points. It could be a promising new way of encoding physical data, allowing for the indication of similar or different clusters, create multiple 'dimensions' or categories, and allow for people to perform interactions between these dimensions by using rotation.

Our findings enable new opportunities for designing systems whose focus is not solely on linear manipulation. The rotation of data objects could allow for novel interactions with data representations. For example, rotating objects to put more or less emphasis on data points, make distinct dimensions or categories in the data or allow for easier comparisons regardless of perspective. To conclude, future work could further explore how the atomic orientation of data points provides new ways of encoding data in 3D space. For example, dynamic composite physicalizations that allow for a both human intervention and actuation to perform free-form cohesion and separation changes to organize data objects in physical space.

\subsection{Reconfiguration for data presentation and organization in physical space}

One of the key reasons for separating and organizing clusters is for data presentation. The utility of proximity and atomic orientation changes could be different between abstract physicalizations and concrete representations. Therefore, the free organization and presentation in space will not be suitable for all types of physicalization. To give an example, it might be more suitable for the communication of trends over actual data points. Related work on static composite physicalizations that use more expressive shapes $[19,32]$, could benefit from exploring organization in a broader sense and how it can support engagement and reflection.

Further utilizing the physical space for data organization also has implications for the interpretation of data. One example is, if we allow for the free organization of data points using atomic orientation, this might require a new approach to designing axes labeling to make sure that users are still able to read data accurately. Moreover, we need to be sure that one person's changes will be interpreted in the same way by others and how manual reconfiguration will exactly coexist in case of multiple users and/or with for example dynamic datasets.

Looking at an overview of example physicalizations [5] we believe that reconfiguration strategies can impact real-world applications. More concrete work on geographical data, molecular structures and constructive visualizations (see $[5,13]$ ) would benefit from incorporating cohesion and separation mechanisms in the interaction design and spatial layout of their physicalizations. Cohesion and separation could then be used to further communicate patterns, facilitate transformations, and inform data interaction with these physical systems.
For example, cohesion and separation changes could facilitate the tweaking of cluster algorithms in an exploratory manner, based on ad-hoc and on the spot insights from the physicalization. If a researcher argues a data point is more strongly associated with a particular cluster due to their tacit knowledge - more than the visualization indicates - pushing that data point into the appropriate cluster then provides feedback for that algorithm. Allowing for reconfiguration hereby allows the researcher to add weights to the data points based on (interaction with) the physicalization itself. Another example use case would be to use cohesion and separation changes as transformations to the dataset - informing the actuation of the dynamic composite physicalization itself. Pushing objects closer together could result in the physicalization to adapt its scale (e.g. from linear to logarithmic) to accommodate the changed data point and thereby offer a quick and intuitive adaptation of the visualization without altering the underlying data value.

Whereas we observed a variety of reconfigurations among participants, the overarching goals were to (i) improve visual consistency and/or (ii) reduce or eliminate ambiguity in the physicalization. Expanding our findings beyond data analysis and presentation, the physical reconfiguration of information objects poses an interesting design strategy. Perhaps, tangible user interfaces (TUIs) could translate input into preferences and actionable results (e.g. smart home control) or be used as a management tool (e.g. with axes being priority, employees, urgency, etc.). The reconfiguration strategies can inform the composition of interactive elements in a TUI and provide design ideas for intuitive ways of interacting with these elements. To give an example, concepts such as cohesion, separation and rotation can inform TUI themes such as tangible manipulation, spatial interaction, and embodied facilitation [12] and help inform how bodily interactions with physical objects in space should translate to underlying computation. One approach is to, for example, use cohesion and separation to clarify (spatial) relations between functionalities of the TUI elements, before and/or during interacting. The atomic orientation of TUI elements could be further explored as a means to perform rotations, allowing interactions on different 'dimensions'.

\subsection{The future design of static, constructive and dynamic composite data physicalizations}

This study shows one example of using physical space, in a labbased setup, which prompts us that there are many other things that should be examined. This includes improving the usability of data objects with different physical properties than, for example, bar charts and exploring future approaches to reconfiguration in space (degree of manipulability), such as stacking, 360 degrees rotation and shape deformation. We provide a few suggestions about how this new knowledge on reconfiguration could be supported with the three types of composite physicalizations.

Looking at static and constructive composite physicalizations, the data objects or 'buckets' of data could be designed in such a way that they allow for more effective comparison in 3D space. This includes their affordance to be compared with each other on multiple axes, or considering their rotation to create a high degree of encoded complexity when constructing visualizations from scratch. Future work could explore the design of combinations 
of these manipulations to utilize 3D space in physically authoring, analyzing or presenting data representations.

For dynamic composite physicalizations, it could be beneficial to reconsider the current technology implementations and see how actuation plays a role in proximity and atomic orientation changes. In 3D space, data values are not solely communicated in height, but also, for example, by their location, orientation, size, shape, behavior and all this in relation to other data objects and the user(s). Research in the field of physicalization should better understand what type of interactions and what relation between human intervention and system actuation allow the user to most effectively perform data organizations. The way that participants applied cohesion and separation changes in our work partially informs us on how we should implement these in transformations with and actuation of dynamic composite physicalizations. To give an example, dynamic composite physicalizations could be responsive before, during, and after (temporary) changes are made to further facilitate data reorganization and presentation.

\subsection{Limitations}

Our study has some limitations that we outline below. First, our study was conducted with a non-interactive prototype in an experimental lab setting. To avoid recognition bias, we used six designs that were abstract representations of data based on the physical bar chart archetype of physicalizations (such as [9, 24, 33]). Whilst our results generalize over these different exemplars of physicalizations, it is unclear how they translate to other data representations, with different form factors and system implementations.

Second, whereas with 2D visualizations there are many kinds of interactions reported in the literature, our work explored basic reconfiguration strategies, not an exhaustive list of possible interactions. Future work is necessary to operationalize these reconfiguration strategies in the form of 'real' datasets and interactive systems to reveal the implications of context on people's sensemaking, how someone else interprets one person's change and how these manipulations change the underlying data model. However, our results are a first starting point for the design of such follow-up studies and systems.

Third, our analysis is based on final configurations at the end of phase 1 and phase 2, thus only provides two snapshots and does not capture the interaction process. These isolated experimental observations of the actions of participants, do not include participants' behavior and - except for anecdotal quotes - their 'intent'. One example is that some participants placed objects back to their original position at the end of phase 1 , to start phase 2 with 'a clean slate', whereas others would make changes more ad-hoc; and some participants would temporarily make changes to check if they created the result they desired and if not, undo the change. This resonates with the concept of offloading as discussed by Kirsh [20] and Liu et al. [25], that explains that rearrangement of physical objects can serve not only to highlight categories/clusters, but also to simplify choice and/or to prevent us from considering irrelevant alternatives. Future work could study in more depth how these strategies, and changes in strategies, match the participants' sensemaking process.
Fourth, our study uses a non-interactive 'data-agnostic' approach as used in prior work that studied aspects of physicalization in isolation $[14,18,29]$. It is also motivated by work from Elliott et al. [6] which describes experimental methods to study visualizations systematically by breaking them up into specific isolated topics and paradigms. An inherent limitation of this approach and methodology is the removal of context, as we studied reconfiguration independently from data actions with a real dataset. Our apparatus were abstract exemplar physicalizations (similar to [18]) and the tasks were focused on spatial clustering. Hence, the reconfigurations that participants performed in our study had the aim of rearranging data points, rather than transforming them. Future work could study the further implications of context on people's interactions with physicalizations and how cluster reconfiguration could be mapped on data transformations.

\section{CONCLUSION}

This paper reports on the extent to which participants were able to use reconfiguration to reorganize physical data objects within a physicalization. We conducted an experiment with six exemplar physicalizations and asked participants to reorganize them using reconfiguration with or without any restrictions. Our study shows that changes in proximity and atomic orientation are the two main reconfiguration strategies that were used to reorganize physical data objects into distinct clusters. Additionally, we provide a further dissection of these strategies and illustrate how these allowed for the change of cohesion and separation of clusters. With our work, we aim to inform the future design of interactions with dynamic composite physicalizations, which can go beyond the use of the plane and utilize the physical 3D space.

\section{ACKNOWLEDGEMENTS}

We would like to thank all participants for volunteering in our study and the anonymous reviewers for their insightful feedback and suggestions.

\section{REFERENCES}

[1] Jason Alexander, Anne Roudaut, Jürgen Steimle, Kasper Hornbæk, Miguel Bruns Alonso, Sean Follmer, and Timothy Merritt. 2018. Grand Challenges in Shape-Changing Interface Research. In Proceedings of the 2018 CHI Conference on Human Factors in Computing Systems (Montreal QC, Canada) (CHI '18). ACM, New York, NY, USA, 1-14. https://doi.org/10.1145/3173574.3173873

[2] Laura Armstrong and Lawrence E. Marks. 1999. Haptic perception of linear extent. Perception \& Psychophysics 61, 6 (1999), 1211-1226.

[3] Fred Attneave and Malcolm D. Arnoult. 1956. The quantitative study of shape and pattern perception. Psychological bulletin 53, 6 (1956), 452.

[4] David L. Davies and Donald W. Bouldin. 1979. A Cluster Separation Measure. IEEE Transactions on Pattern Analysis and Machine Intelligence PAMI-1, 2 (1979), 224-227. https://doi.org/10.1109/TPAMI.1979.4766909

[5] Pierre Dragicevic and Yvonne Jansen. 2012. List of Physical Visualizations. www.dataphys.org/list. Last accessed Nov 2020.

[6] Madison A. Elliott, Christine Nothelfer, Cindy Xiong, and Danielle Albers Szafir. 2020. A Design Space of Vision Science Methods for Visualization Research. IEEE Transactions on Visualization and Computer Graphics (2020). https://doi.org/10. 1109/TVCG.2020.3029413

[7] Aluna Everitt, Faisal Taher, and Jason Alexander. 2016. ShapeCanvas: An Exploration of Shape-Changing Content Generation by Members of the Public. In Proceedings of the 2016 CHI Conference on Human Factors in Computing Systems (San Jose, California, USA) (CHI '16). ACM, New York, NY, USA, 2778-2782. https://doi.org/10.1145/2858036.2858316

[8] Danyang Fan, Alexa Fay Siu, Sile O'Modhrain, and Sean Follmer. 2020. Constructive Visualization to Inform the Design and Exploration of Tactile Data 
Representations. In The 22nd International ACM SIGACCESS Conference on Computers and Accessibility (Virtual Event, Greece) (ASSETS '20). ACM, New York, NY, USA, Article 60, 4 pages. https://doi.org/10.1145/3373625.3418027

[9] Sean Follmer, Daniel Leithinger, Alex Olwal, Akimitsu Hogge, and Hiroshi Ishii. 2013. InFORM: Dynamic Physical Affordances and Constraints through Shape and Object Actuation. In Proceedings of the 26th Annual ACM Symposium on User Interface Software and Technology (St. Andrews, Scotland, United Kingdom) (UIST '13). ACM, New York, NY, USA, 417-426. https://doi.org/10.1145/2501988.2502032

[10] Pauline Gourlet and Thierry Dassé. 2017. Cairn: A Tangible Apparatus for Situated Data Collection, Visualization and Analysis. In Proceedings of the 2017 Conference on Designing Interactive Systems (Edinburgh, United Kingdom) (DIS '17). ACM, New York, NY, USA, 247-258. https://doi.org/10.1145/3064663.3064794

[11] Jeffrey Heer and Michael Bostock. 2010. Crowdsourcing Graphical Perception Using Mechanical Turk to Assess Visualization Design. In Proceedings of the SIGCHI Conference on Human Factors in Computing Systems (Atlanta, Georgia, USA) (CHI '10). ACM, New York, NY, USA, 203-212. https://doi.org/10.1145/ 1753326.1753357

[12] Eva Hornecker and Jacob Buur. 2006. Getting a Grip on Tangible Interaction: A Framework on Physical Space and Social Interaction. In Proceedings of the SIGCHI Conference on Human Factors in Computing Systems (Montréal, Québec, Canada) (CHI '06). ACM, New York, NY, USA, 437-446. https://doi.org/10.1145/1124772. 1124838

[13] Samuel Huron, Sheelagh Carpendale, Alice Thudt, Anthony Tang, and Michael Mauerer. 2014. Constructive Visualization. In Proceedings of the 2014 Conference on Designing Interactive Systems (Vancouver, BC, Canada) (DIS '14). ACM, New York, NY, USA, 433-442. https://doi.org/10.1145/2598510.2598566

[14] Samuel Huron, Yvonne Jansen, and Sheelagh Carpendale. 2014. Construct ing Visual Representations: Investigating the Use of Tangible Tokens. IEEE Transactions on Visualization and Computer Graphics 20, 12 (2014), 2102-2111. https://doi.org/10.1109/TVCG.2014.2346292

[15] Yvonne Jansen and Pierre Dragicevic. 2013. An Interaction Model for Visualizations Beyond The Desktop. IEEE Transactions on Visualization and Computer Graphics 19, 12 (2013), 2396-2405. https://doi.org/10.1109/TVCG.2013.134

[16] Yvonne Jansen, Pierre Dragicevic, and Jean-Daniel Fekete. 2013. Evaluating the Efficiency of Physical Visualizations. In Proceedings of the SIGCHI Conference on Human Factors in Computing Systems (Paris, France) (CHI '13). ACM, New York, NY, USA, 2593-2602. https://doi.org/10.1145/2470654.2481359

[17] Yvonne Jansen, Pierre Dragicevic, Petra Isenberg, Jason Alexander, Abhijit Karnik, Johan Kildal, Sriram Subramanian, and Kasper Hornbæk. 2015. Opportunities and Challenges for Data Physicalization. In Proceedings of the 33rd Annual ACM Conference on Human Factors in Computing Systems (Seoul, Republic of Korea) (CHI '15). ACM, New York, NY, USA, 3227-3236. https://doi.org/10.1145/2702123. 2702180

[18] Yvonne Jansen and Kasper Hornbæk. 2016. A Psychophysical Investigation of Size as a Physical Variable. IEEE Transactions on Visualization and Computer Graphics 22, 1 (2016), 479-488. https://doi.org/10.1109/TVCG.2015.2467951

[19] Rohit Ashok Khot, Jeewon Lee, Larissa Hjorth, and Florian 'Floyd' Mueller. 2014 SweatAtoms: Understanding Physical Activity through Material Artifacts. In CHI '14 Extended Abstracts on Human Factors in Computing Systems (Toronto, Ontario, Canada) (CHI EA '14). ACM, New York, NY, USA, 173-174. https: //doi.org/10.1145/2559206.2579479

[20] David Kirsh. 1995. The Intelligent Use of Space. Artificial Intelligence 73, 1-2 (1995), 31-68. https://doi.org/10.1016/0004-3702(94)00017-U

[21] Kurt Koffka. 2013. Principles of Gestalt Psychology. The International Library of Psychology, Vol. 44. Routledge.

[22] Mathieu Le Goc, Lawrence H. Kim, Ali Parsaei, Jean-Daniel Fekete, Pierre Drag icevic, and Sean Follmer. 2016. Zooids: Building Blocks for Swarm User Interfaces. In Proceedings of the 29th Annual Symposium on User Interface Software and Technology (Tokyo, Japan) (UIST '16). ACM, New York, NY, USA, 97-109. https://doi.org/10.1145/2984511.2984547

[23] Mathieu Le Goc, Charles Perin, Sean Follmer, Jean-Daniel Fekete, and Pierre Dragicevic. 2018. Dynamic Composite Data Physicalization Using Wheeled Micro-Robots. IEEE Transactions on Visualization and Computer Graphics 25, 1 (2018), 737-747. https://doi.org/10.1109/TVCG.2018.2865159

[24] Daniel Leithinger and Hiroshi Ishii. 2010. Relief: A Scalable Actuated Shape Display. In Proceedings of the Fourth International Conference on Tangible, Embedded, and Embodied Interaction (Cambridge, Massachusetts, USA) (TEI '10). ACM, New York, NY, USA, 221-222. https://doi.org/10.1145/1709886.1709928

[25] Zhicheng Liu and John Stasko. 2010. Mental Models, Visual Reasoning and Interaction in Information Visualization: A Top-down Perspective. IEEE Transactions on Visualization and Computer Graphics 16, 6 (2010), 999-1008. https: //doi.org/10.1109/TVCG.2010.177

[26] Deborah Lupton. 2017. Feeling your data: Touch and making sense of personal digital data. New Media \& Society 19, 10 (2017), 1599-1614.

[27] Tamara Munzner. 2014. Visualization Analysis and Design. CRC Press, Boca Raton FL, United States.

[28] Majken K. Rasmussen, Esben W. Pedersen, Marianne G. Petersen, and Kasper Hornbæk. 2012. Shape-Changing Interfaces: A Review of the Design Space and
Open Research Questions. In Proceedings of the SIGCHI Conference on Human Factors in Computing Systems (Austin, Texas, USA) (CHI'12). ACM, New York, NY, USA, 735-744. https://doi.org/10.1145/2207676.2207781

[29] Kim Sauvé, Dominic Potts, Jason Alexander, and Steven Houben. 2020. A Change of Perspective: How User Orientation Influences the Perception of Physicalizations. In Proceedings of the 2020 CHI Conference on Human Factors in Computing Systems (Honolulu, HI, USA) (CHI '20). ACM, New York, NY, USA, 1-12. https://doi.org/10.1145/3313831.3376312

[30] Stephen Smart and Danielle Albers Szafir. 2019. Measuring the Separability of Shape, Size, and Color in Scatterplots. In Proceedings of the 2019 CHI Conference on Human Factors in Computing Systems (Glasgow, Scotland Uk) (CHI '19). ACM, New York, NY, USA, 1-14. https://doi.org/10.1145/3290605.3300899

[31] Miriam Sturdee, John Hardy, Nick Dunn, and Jason Alexander. 2015. A Public Ideation of Shape-Changing Applications. In Proceedings of the 2015 International Conference on Interactive Tabletops \& Surfaces (Madeira, Portugal) (ITS '15). ACM, New York, NY, USA, 219-228. https://doi.org/10.1145/2817721.2817734

[32] Simon Stusak, Aurélien Tabard, Franziska Sauka, Rohit Ashok Khot, and Andreas Butz. 2014. Activity Sculptures: Exploring the Impact of Physical Visualizations on Running Activity. IEEE Transactions on Visualization and Computer Graphics 20, 12 (2014), 2201-2210. https://doi.org/10.1109/TVCG.2014.2352953

[33] Faisal Taher, John Hardy, Abhijit Karnik, Christian Weichel, Yvonne Jansen, Kasper Hornbæk, and Jason Alexander. 2015. Exploring Interactions with Physically Dynamic Bar Charts. In Proceedings of the 33rd Annual ACM Conference on Human Factors in Computing Systems (Seoul, Republic of Korea) (CHI '15). ACM, New York, NY, USA, 3237-3246. https://doi.org/10.1145/2702123.2702604

[34] Faisal Taher, Yvonne Jansen, Jonathan Woodruff, John Hardy, Kasper Hornbæk, and Jason Alexander. 2017. Investigating the Use of a Dynamic Physical Bar Chart for Data Exploration and Presentation. IEEE Transactions on Visualization and Computer Graphics 23, 1 (2017), 451-460. https://doi.org/10.1109/TVCG.2016. 2598498

[35] Alice Thudt, Uta Hinrichs, Samuel Huron, and Sheelagh Carpendale. 2018. SelfReflection and Personal Physicalization Construction. In Proceedings of the 2018 CHI Conference on Human Factors in Computing Systems (Montreal QC, Canada) (CHI '18). ACM, New York, NY, USA, 1-13. https://doi.org/10.1145/3173574. 3173728

[36] Colin Ware. 2019. Information Visualization: Perception for Design. Morgan Kaufmann, Cambridge MA, United States.

[37] Tiffany Wun, Jennifer Payne, Samuel Huron, and Sheelagh Carpendale. 2016. Comparing Bar Chart Authoring with Microsoft Excel and Tangible Tiles. Computer Graphics Forum 35, 3 (2016), 111-120. https://doi.org/10.1111/cgf.12887 arXiv:https://onlinelibrary.wiley.com/doi/pdf/10.1111/cgf.12887 